Mediterránea Ser. Biol. (1989), n. ${ }^{\circ}$ 11. Pág. 77-120

\title{
ANÁLISIS DE LA DISTRIBUCIÓN DE LA AVIFAUNA NIDIFICANTE EN LA PROVINCIA DE ALICANTE
}

\author{
por \\ J. A. GIL-DELGADO ${ }^{1}$, G. LÓPEZ ${ }^{2}$, L. RICO $^{3}$, \\ M. I. SIRVENT ${ }^{4}$, J. VILLAPLANA $^{5}$, L. ALBENTOSA ${ }^{6}$
}

\section{RESUMEN}

El número de especies de aves observadas durante la estación de nidificación en la provincia de Alicante es de 151, de las cuales 146 han nidificado con seguridad en el último decenio. En relación con otras regiones ibéricas la avifauna alicantina está empobrecida. Este patrón se observa también a nivel intraprovincial mediante una disminución del número de especies de norte a sur y de oeste a este. Los enclaves palustres del sureste de Alicante rompen la tendencia. Las zonas más pobres en especies son las regiones costeras, coincidentes con las áreas más antropizadas. Este suceso sugiere que parte de la pérdida de especies está provocada por la acción del hombre. No obstante, factores de indole biogeográfica (situación costera, efecto peninsular etc...) resultan básicos para comprender el empobrecimiento de especies.

\section{SUMMARY}

The number of bird species observed in Alicante province (SE Spain) during the breeding season was 151 , reproduction being proved in 146, at least in some years. Compared with other Iberian regions, the breeding avifauna of Alicante is impoverished. Within this province the number of breeding bird species decreases from North to South and from West to East, but the marshes and saltmarshes of the south break this trend. The poorer area in bird species is the coastal strip, specially where the influence of man is stronger. But, although the man action seems an important factor in determining species number, other biogeographical factors, such as peninsular effect or coastal situation, may be also relevant.

(1) Dpto. Ecología, Facultad de Biológicas, Universidad de Valencia. Burjassot (Valencia).

(2) Dpto. Ciencias Ambientales y Recursos Naturales. Universidad de Alicante (Alicante).

(3) Calle Genaro Candela, 10. Aspe (Alicante).

(4) Calle Marqués de Molins, 2. (Alicante).

(5) Calle 9 de Octubre, 21. Gandía (Valencia).

(6) Avda. Estación, 17. (Alicante). 


\section{INTRODUCCIÓN}

En general los estudios sobre la avifauna de áreas geográficas más o menos extensas tienden a describir, mediante cartografía, la distribución de cada una de las especies que nidifican en el país, región o comarca estudiada. El resultado es una recopilación de mapas y cortos artículos monoespecíficos que eluden otros tipos de análisis. Por este esquema se rigen gran parte de los trabajos realizados hasta el momento (p. e., Sharrock, 1976; Muntaner, et al., 1983; Elósegui, 1985).

Alternativamente y con los mismos datos básicos se puede obtener una visión más sintética de la distribución de la avifauna y, en función de ésta, caracterizar diferentes subregiones de la superficie estudiada. Thomas (1979) sigue esta vía al analizar la avifauna de la isla de Tasmania para clasificar los distintos hábitats insulares en función de sus componentes orníticos.

En el presente artículo, aunque está basado en la cartografía de cada una de las especies que nidifican en Alicante, pretendemos ajustarnos a la segunda vía, explorando como se agrupan las especies que nidifican en Alicante y cómo se segrega el territorio alicantino en función de sus componentes orníticos.

\section{ÁREA DE ESTUdio}

La provincia de Alicante tiene una extensión de $5.863 \mathrm{~km}^{2}$ y está situada a orillas del mar Mediterráneo en el sector oriental de la Península Ibérica (Figura 1).



Figura 1.-Situación de la provincia de Alicante en la Península Ibérica. 




Figura 2.-Medio físico de la provincia de Alicante. a) Mapa del relieve, ríos y principales zonas húmedas. Línea de puntos: curva de nivel de $400 \mathrm{~m}$. Línea continua: curva de nivel de $800 \mathrm{~m}$. Zonas húmedas: 1.-Hondo de Elche. 2.-Salinas de Santa Pola. 3. - Laguna de la Mata. 4.-Salinas de Torrevieja. b) Mapa de isotermas (según A.López, et al., 1978). c) Lluvias mediasanuales (según A. López, et al., 1978). d) Distribución del medio forestal (Ministerio de Agricultura, Pesca y Alimentación 1986). 
La Figura 2 muestra los mapas de relieve (2a) y los correspondientes a las temperaturas ( $2 \mathrm{~b}$ ) y precipitaciones medias anuales $(2 \mathrm{c})$. El relieve es accidentado, con la excepción del sector sur-sureste en donde se localizan las zonas húmedas del Hondo de Elche y el curso bajo del río Segura. Las precipitaciones presentan dos máximos, primavera y otoño, siendo octubre el mes más lluvioso (más de $601 / \mathrm{m}^{2}$ ). La temperatura es benigna y el mes más frío, enero, presenta temperaturas medias de $10^{\circ}-12^{\circ} \mathrm{C}$. en la franja costera y de $5^{\circ}-7^{\circ} \mathrm{C}$. en las zonas del interior. Estas últimas áreas registran heladas ocasionales. Los veranos son secos y calurosos. Información más detallada de estos aspectos se puede obtener en Castillo y Ruiz (1977).

La vegetación está formada por un mosaico de cultivos, eriales, zonas de matorral con estructuras diferentes y retazos de bosques naturales o procedentes de trabajos de repoblación. La Figura $2 \mathrm{~d}$ muestra la distribución del pino carrasco (Pinus halepensis), que es la especie arbórea más abundante, según la cartografía del Ministerio de Agricultura, Pesca y Alimentación (1986). Al estar los encinares intercalados entre los pinares este mapa representa satisfactoriamente la distribución del hábitat forestal en la provincia, pese a que en tiempos recientes se han registrado alteraciones a consecuencia de los incendios.

Los cultivos cubren el $53,4 \%$ de la superficie provincial. Entre ellos los más extendidos son los de secano con los almendros $(9,6 \%)$ y viñedos $(9,1 \%)$ abarcando superficies más amplias (véanse mapas en Ministerio de Agricultura, Pesca y Alimentación, 1986).

\section{MATERIAL Y MÉTODO}

La unidad de muestreo es la cuadrícula de $10 \times 10 \mathrm{~km}$. de la red UTM. Este tamaño es el más utilizado en estudios de esta índole (p. e., Sharrock, 1976; Muntaner, et al., 1983). Cada cuadrícula se visitó al menos en tres ocasiones durante las temporadas reproductoras de 1984 y 1985 . No obstante, existe una importante adición de observaciones procedentes de temporadas anteriores y posteriores a las de los años señalados.

Las especies observadas en cada cuadrícula se clasificaron de acuerdo con las tres categorías clásicas en relación con el grado de certidumbre sobre la nidificación en la cuadrícula: nidificación segura, probable y posible. Información sobre el procedimiento para asignar las especies a una de las tres categorías se puede encontrar en Sharrock (1976) y Muntaner et al. (1983) entre otros.

El área de estudio se dividió en tres sectores, cada uno de ellos explorado por una pareja de entre el equipo de autores. Los sectores y los observadores se corresponden con el tercio norte (JAGD y JV), y el resto de la provincia repartido en un sector oriental (GL y LA) y un sector occidental (LR y MIS).

La representación cartográfica de cada una de las categorías se muestra al principio del Apéndice I, en donde están registrados los mapas correspondientes a cada una de las especies que nidifican en Alicante. Sin embargo, debemos apuntar dos consideraciones: a) las especies raras no están representadas (dos especies), y b) las especies caracterizadas por ocupar amplios territorios (p.e, aves de presa), muestran en sus mapas correspondientes las cuadrículas en las que poseen porciones de territorio, significando que no en todas las cuadrículas puntuadas nidifican, de manera que encubren las cuadrículas con nidificación segura. 
Para determinar la segregación de la provincia de Alicante en función de sus componentes orníticos se ha calculado la matriz de similitud entre cuadriculas $10 \times 10$ mediante el índice de Jacquard (véase Margalef, 1974), y las especies se han clasificado a partir de la matriz de los coeficientes de correlación (véase Legendre y Legendre, 1979). Para generar el mapa de isolíneas con igual número de especies nidificantes (Figura 7) se ha utilizado el programa Golden Graphics System (Golden Software Inc., 1985) mediante el algoritmo Kriging. Para aplicar el programa asumimos que el número de especies encontrado en cada cuadrícula de $10 \times 10 \mathrm{~km}$. se encuentran en un punto situado en su centro. Lógicamente el número de especies es variable dentro de una misma cuadrícula, por lo que las isolíneas pretenden tan sólo mostrar el gradiente de variación a nivel provincial.

\section{RESULTADOS}

El número de especies de aves, pertenecientes a alguna de las tres categorías, observadas en la provincia de Alicante es de 151 . De ellas, 146 nidifican con seguridad, y las 5 restantes, de acuerdo con las normas establecidas en este tipo de estudios (véase Sharrock, 1976), pertenecen a la categoría de probables (3) y posibles (2). En el apéndice I se muestran los mapas de distribución de cada una de las especies. La isla de Tabarca no está representada en los ma-



Figura 3.-Número de especies por cuadrícula $10 \times 10 \mathrm{~km}$. en Alicante. 




TABLA I.-Relación de especies nidificantes en la isla de Tabarca. $1=$ Nidificante seguro. $3=$ Nidificante posible. $+=$ Nidifica en el continente. $-=$ No encontrado en el continente.

pas, pero las especies que nidifican en la isla se muestran en la Tabla I. Entre las especies que crían en Tabarca sólo el paíño común (Hydrobates pelagicus) no ha sido encontrado en el área continental.

Entre las especies nidificantes, el avefría (Vanellus vanellus) ha nidificado en una temporada; el tarro blanco (Tadorna tadorna) es un colonizador reciente (Calvo e Iborra 1986); el águila pescadora (Pandion haliaetus) y el cormorán moñudo (Phalacrocorax aristotelis) han dejado de nidificar en tiempos recientes, aunque mantienen individuos adultos en Alicante durante la época de cría.

El número de especies que cría por cuadrícula varía entre 30 y 86 (Figura 3). Las zonas de Sierra de Salinas, Sierra de Mariola, Font Roja y las áreas húmedas del Hondo de Elche son las que acogen a mayor número de especies, mientras que las zonas costeras son las que presentan una mayor pobreza ornítica (véase Figura 3).

\section{Segregación de la provincia de Alicante en función de la avifauna}

La Figura 4 muestra cómo se segrega la superficie alicantina en función de sus componentes orníticos. Considerando como límite el valor de similitud de 0.6 , la provincia de Alicante se segrega en once grupos que abarcan superficies comprendidas entre los 200 y los $1.500 \mathrm{~km}^{2}$. Además cuatro cuadrículas se fusionan a diversos grupos por debajo del nivel considerado. Estas cuadrículas coinciden con áreas con un alto grado de antropización, o bien se corresponden con zonas húmedas alteradas (cuadrículas representadas por estrellas blancas en la Figura 4) y es con las cuadrículas en donde se insertan las zonas húmedas (estrellas negras) con las que se agrupan en primer lugar (véanse Figuras 4 y 5). La fusión de los grupos por debajo del nivel 0.6 se muestra en la Figura 5.

La Tabla II presenta el número medio de especies que nidifican en cada uno de los once grupos en los que se segrega la provincia.

El grupo I se extiende por $1.200 \mathrm{~km}^{2}$ en el sector noreste de Alicante y acoge a una cuadrícula costera que está fuertemente influida por las Morras de Benitachell, área con un matorral bien conservado, y que se extiende desde la Punta de Moraira a la Cala de la Granadella. Estas cuadrículas están formadas por un terreno muy quebrado, desforestado, y en donde los barrancos, más húmedos y con una mayor cobertura vegetal, sirven de refugio a un buen número de especies.

El grupo II lo constituyen $400 \mathrm{~km}^{2}$ que engloban a la Font Roja y a la Sierra de Mariola. El área presenta superficies forestadas que sirven de refu- 




Figura 4.- Segregación de la provincia de Alicante en función de sus componentes orníticos. Signos idénticos agrupan cuadrículas similares. Algunos signos están representados en una sola cuadrícula; su configuración permite apreciar con qué grupo se fusionan antes. Así, las estrellas blancas, grande y pequeña, se agrupan en primer lugar con los grupos definidos por estrellas negras. Para completar la información debe consultarse la Figura 5.

Grupo I

Grupo II

Grupo III

Grupo IV

Grupo V

Grupo VI

Grupo VII

Grupo VIII

Grupo IX

Grupo X

Grupo XI $\bar{x}$ ESPECIES

55.1

61.7

66.6

47.6

67.5

38.2

41.6

58

39

61.7

49
S.D.

6.6

7.05

7.7

4.1

2

3.6

1.7

4

2

11.3

6.98

\begin{tabular}{r}
$\mathrm{n}$ \\
\hline 12 \\
4 \\
15 \\
7 \\
2 \\
4 \\
5 \\
2 \\
2 \\
4 \\
5
\end{tabular}

TABLA II.-Número de especies para cada uno de los once grupos en que se segrega la provincia de Alicante. Los signos que identifican a cada grupo se corresponden con los de la figura 4. 




Figura 5.-Agrupación de los once grupos en que se divide la provincia de Alicante a partir del nivel 0.6. Las cuadrículas que se reúnen por encima de este nivel están representadas por el mismo signo en la Figura 4.

gio a un pequeño número de especies de abolengo forestal, y que únicamente se encuentran en esta región y en la Sierra de Salinas.

El grupo III es el que se extiende por una superficie más amplia, 1.500 $\mathrm{km}^{2}$, abarcando la comarca del Alto Vinalopó y los términos de Castalla y Tibi en el centro de la provincia. Este grupo es el que presenta por término medio el mayor número de especies por cuadrícula (Tabla II), con la excepción de las dos cuadrículas que forman el grupo $\mathrm{V}$ y que se encuentran en la frontera entre la comarca del Alto Vinalopó y los humedales del Hondo de Elche.

El grupo IV está formado por 7 cuadrículas, $700 \mathrm{~km}^{2}$, segregadas en tres bloques discontinuos a nivel geográfico (véanse Figuras 4 y 5). Estas cuadrículas son regiones de frontera y que se caracterizan por la pérdida de especies respecto a los grupos II y III.

El grupo $\mathrm{V}$ se ha comentado al tratar el grupo III.

El grupo VI es el más pobre en especies (Tabla II) y acoge una superficie de 300 a $400 \mathrm{~km}^{2}$ que se corresponden con las cuadrículas costeras de la mitad septentrional de la provincia. En estas cuadrículas se sitúan los grandes centros turísticos alicantinos.

El grupo VII lo forman cinco cuadrículas cercanas a la costa y con un número medio de especies similar al del grupo anterior.

Los grupos VIII y IX apenas sobrepasan los $100 \mathrm{~km}^{2}$, pero mientras el grupo IX define los eriales y cultivos cerealísticos del noroeste de Alicante, el grupo VIII hay que observarlo con ciertas precauciones, pues una exploración más detallada de sus cuadrículas (los sectores correspondientes a otras provincias no se visitaron) pudiera hacer posible su agregación al grupo III.

Los grupos X y XI se extienden aproximadamente por $400 \mathrm{~km}^{2}$ cada uno de ellos. Los dos grupos representan a las zonas húmedas alicantinas, y se puede apreciar una mayor riqueza en el grupo X (zonas húmedas de Santa Pola y el Hondo de Elche) que en el grupo XI (Laguna de La Mata y Salinas de Torrevieja) (véase Tabla II). 


\section{Segregación de las especies de aves que nidifican en Alicante}

La Figura 6 muestra el dendrograma resultante de la matriz de correlación entre las diferentes especies a partir de los valores de presencia o ausencia de las 151 especies en cada una de las cuadrículas. A causa del elevado número de especies, sólo presentamos en el dendrograma las fusiones por debajo del nivel 0.7 , y es en el apéndice II en donde se presentan las especies que componen cada agregación por encima del nivel mencionado.

Considerando como límite el valor de 0.6 , las aves alicantinas se segregan en once grupos que incluyen entre 2 y 45 especies. La Tabla III muestra para cada uno de los once grupos el número de especies pertenecientes a cada una de las categorías ornitogeográficas de Voous (1960).

El grupo I es el que integra mayor número de especies (45), de ellas 39 son típicas de las zonas húmedas. La carraca (Coracias garrulus), integrada en este grupo, es una especie muy rara en Alicante y su fusión con el grupo tiene lugar por debajo del nivel de 0.6, además las especies escasas presentan próblemas de interpretación en este tipo de análisis (véase Margalef, 1974).

El grupo II está compuesto por especies que presentan en Alicante una distribución que abarca la totalidad de la provincia. Son especies capaces de aceptar hábitats antrópicos o zonas dominadas por el matorral. El grupo está compuesto por especies de amplia distribución en Europa (Paleárticas y Euroturquestanas), junto a un buen número de especies con distribución mediterránea (véase Tabla III).

El grupo III está formado por especies que en gran medida dependen del medio forestal, por tanto, recluidas en aquellas cuadrículas que conservan superficies arboladas y que en general están localizadas en el tercio norte de Alicante. El grupo incluye especies muy localizadas (p.e., Erithacus rubecula). Las categorías dominantes en este grupo son la Paléartica y la Europea, que en conjunto constituyen más del $60 \%$ de las especies. Estos resultados concuerdan con los de varios autores (p.e., Tellería, 1980; Potti, 1985) sobre el aumento de la importancia de las aves Paleárticas y Europeas según avanza la sucesión hacia el bosque.

El grupo IV incluye especies que residen en áreas de montaña, e incluye la única especie Paleomontana de Alicante, la chova piquirroja (Pyrrhocorax pyrrhocorax), y dos especies Paleoxeromontanas. La presencia en este grupo de especies como el ruiseñor común (Luscinia megarhynchos) y el zarcero común (Hippolais polyglotta), que no encajan en esta descripción, guarda relación con el uso que hacen del lecho de los barrancos, y que coinciden a nivel geográfico con las sierras que separan.

El grupo V está formado por dos especies muy localizadas y con distribución dispersa, básicamente en la mitad norte de la provincia.

El grupo VI integra especies que se distribuyen por la mitad occidental de Alicante y que faltan o son muy escasas en áreas cercanas a la costa. Junto a ellas aparecen especies con distribución dispersa.

Los restantes grupos están compuestos por un reducido número de especies que se caracterizan por residir en hábitats muy concretos o ser raras en el ámbito provincial. Así, los grupos VII y VIII están formados por especies muy raras o de distribución irregular; el grupo IX agrega las especies de áreas abiertas; el grupo X, especies costeras; y el grupo XI, dos especies que están ligadas a los cursos fluviales y que se restringen al norte de la provincia. 









Grupo



Figura 6.-Dendrograma que muestra la formación de varios grupos de especies de aves en función de su área de distribución en la provincia de Alicante. El número al final de cada rama corresponde al de especies incluidas en ella por encima del nivel de 0.7. Las especies que componen cada rama y cada grupo aparecen detalladas en el apéndice II. 


\section{DISCUSIÓN}

La avifauna alicantina se segrega en once grupos, de los cuales cinco agrupan más de 15 especies. De estos grupos mayores sólo dos presentan una distribución amplia en la provincia, y están constituidos por especies que aceptan de buen grado las áreas antrópicas, que se adecúan bien a superficies degradadas, o que disponen de hábitats que se extienden por amplias superficies en Alicante. Los otros tres grupos se establecen en áreas muy determinadas; es el caso de las especies forestales, de las especies que residen en zonas húmedas y de las especies que no se encuentran en la franja costera alicantina. Entre estos tres grupos es precisamente el que agrega las especies de aves asociadas a zonas húmedas el más numeroso, y sin embargo, con la mayor parte de las especies que lo componen recluidas en el sureste de Alicante. El segundo grupo, al que pertenecen las especies con marcada antropofilia, acoge las especies con distribución más amplia, y que en algunas cuadrículas constituyen la casi totalidad de la avifauna nidificante. Es predecible que en el futuro este grupo se incremente mediante la asociación definitiva al mismo del estornino negro (Sturnus unicolor), especie antropófila y en proceso de expansión.

La Figura 5 se puede simplificar por agregación de los grupos más similares, quedando Alicante dividida en cuatro regiones. La primera recorre el norte de la provincia desde las Morras de Benitachell hacia el oeste y con tendencia al incremento en el número de especies según nos alejamos de las zonas costeras, y que desde el sur de Villena abarca el cuadrante noroccidental, acogiendo la comarca del Alto Vinalopó (GI, GII y GIII); en este gran grupo el incremento es más notorio en aquellas cuadrículas que presentan superficies forestales. La segunda región es fronteriza con la primera y recorre la provincia desde el noreste al suroeste, separando la primera de la tercera región, la cual se corresponde con la franja costera de la mitad septentrional de la provincia. La cuarta región, rica en especies, incluye las zonas húmedas. La Figura 7 muestra los gradientes de variación del número de especies de aves en Alicante. El que las escasas superficies forestales se encuentren en los sectores norte y occidental de la provincia, al tiempo que existen especies que no se encuentran en las inmediaciones de la costa, origina que las zonas del interior sean más ricas en especies orníticas, pudiéndose apreciar un descenso en el número de especies de norte a sur y de oeste a este. Las zonas húmedas constituyen una excepción.

Por otra parte, si observamos la Figura 2a se puede apreciar que a grandes rasgos las fronteras entre las dos primeras regiones coincide con la curva de nivel de $\operatorname{los} 400 \mathrm{~m}$. de altitud sobre el nivel del mar (véanse Figuras 2a y 4). La reducción de especies al este de dicha curva de nivel coincide con el área en donde están establecidos los mayores complejos turísticos y la ciudad de Alicante. El que la cuadrícula costera en donde se encuadran las Morras de Benitachell (61 especies) tenga más conexión con las áreas del interior que con las restantes cuadrículas de la segunda y tercera región, permite achacar al impacto humano parte de la reducción del número de especies en las zonas costeras, ya que otros ecosistemas costeros similares (p.e., Mongó, Sierra Helada), aunque más degradados, presentan menor número de especies. Más evidencia de este proceso podemos extraer de la cuadrícula situada al este de Mariola (45 especies), y en la que se encuentran los centros urbanos de Alcoy, Muro 




Figura 7.-Mapa de isolíneas con igual número de especies nidificantes, realizado a partir de los datos que se muestran en la figura 3 (ver texto).

de Alcoy y Cocentaina, agregada al grupo IV con otras cuadrículas con cierto grado de antropización (cultivos, urbanizaciones).

La provincia de Tarragona (152 especies, véase Muntaner et al., 1983), montañosa y con zonas húmedas (Delta del Ebro), es equiparable a Alicante por sus dimensiones. Ambas provincias tienen un número de especies nidificantes similar. Sin embargo, Gerona (véase Muntaner et al. 1983) y Navarra (véase Elósegui, 1985) mantienen un número de especies superior, de tal manera que, respecto a ellas, Alicante muestra una fauna ornítica empobrecida. De acuerdo con el criterio de Cheylan y Cheylan (1976) el cociente entre especies norteñas (Holárticas, Paleárticas, Euroturquestanas, Europeas, Paleomontanas) y mediterráneas (Mediterráneas, Turquestano-mediterránea, Viejo Mundo, Paleoxeromontanas, Paleoxéricas, Indoafricanas) se sitúa entre 1.3 y 1.4 para el conjunto de la avifauna nidificante en Alicante y para la mayoría de los grupos de especies que aparecen en la Tabla III. Solamente dos grupos se desvían de este patrón. El grupo III, formado por especies forestales, que muestra un cociente de 5.3 por la mayor proporción de especies norteñas, y que apoya el papel de los bosques como medio de penetración de especies norteñas (Blondel, 1981; Tellería, 1980 y 1987; Potti, 1985). El otro es el grupo IX, que presenta un cociente de 0.6 , lo que indica un claro dominio de las especies mediterráneas, situación inversa a la del resto de los grupos. Aquí se incluyen especies cuya área de distribución comprende principalmente el ángulo noroeste 
de la provincia, donde el paisaje se asemeja a las áreas seudoesteparias de La Mancha. Todas las especies mediterráneas de este grupo, excepto Otus scops, son propias de este medio. La situación es inversa a la de los bosques, de forma que las estepas ibéricas podrían ser un medio de penetración de especies mediterráneas.

El cociente entre especies norteñas y mediterráneas para la avifauna alicantina es de 1.4, menor que el que se obtiene para el conjunto de la avifauna que nidifica en la Pehínsula Ibérica, que es de 2. Esto indica que la avifauna alicantina está empobrecida de especies norteñas. De las 68 especies norteñas ibéricas que faltan en Alicante, la situación geográfica de esta provincia es causa de la ausencia de 46 especies, al no alcanzar su distribución latitudes tan bajas. De las 22 especies norteñas restantes, 11 son especies forestales, por lo que su ausencia en Alicante tendría relación con la baja extensión de territorio ocupado por bosques, muchos de ellos de baja calidad para la fauna (repoblaciones monoespecificas de pinos). La falta de otras 5 especies puede explicarse por la carencia de recursos y la falta o degradación de los hábitats que necesitan (Aquila heliaca, Gyps Fulvus, Ardea cinerea, Cinclus cinclus y Prunella modularis).

En Alicante faltan 17 especies de aves mediterráneas. De ellas, 5 son aves de presa cuya ausencia se explicaría en función de la degradación de los hábitats. Otras 6 especies ausentes en la actualidad presentan una distribución muy restringida en la Península (p.e., Aythya nyroca, Porphyrio porphyrio).

Otros factores de índole geográfica deben influir en el empobrecimiento de especies nidificantes en Alicante. En general, las áreas periféricas de los continentes tienden a tener menor número de especies por su alejamiento de las zonas de aprovisionamiento de especies (Blondel, 1985). Esta situación se acentúa en las penínsulas, en algunas de las cuales se ha observado una disminución en su riqueza de especies (MacArthur y Wilson, 1967). La provincia de Alicante encaja de lleno en este esquema, y aunque los factores biogeográficos apuntados deben de influir en el empobrecimiento de especies, parte del empobrecimiento en especies debe achacarse a la acción humana. El menor número de especies en las cuadrículas más antropizadas evidencian este último efecto.

\section{AGRADECIMIENTOS}

Para la realización de este trabajo se ha contado con la ayuda de un nutrido grupo de personas a las que deseamos expresar nuestro agradecimiento. En el campo nos acompañaron en frecuentes ocasiones Manolo Carrascosa, Juan Antonio Gómez Picazo, Alfredo Linares, Salvador Peiró, Ángel Sánchez, Antonio Vidal y Toni Zaragozí. Generosamente cedieron información inédita sobre la avifauna alicantina José Julián Rico Cerdá, José Damián Navarro Medina y Antonio Sánchez. María del Carmen Catalá colaboró en la pesada tarea de realizar los mapas. Para la realización de este trabajo se contó con una ayuda del Instituto de Estudios Alicantinos de la Excma. Diputación Provincial (hoy Instituto Juan Gil-Albert). 


\section{BIBLIOGRAFÍA}

BLONDEL, J. (1981). Structure and dynamics of bird communities in Mediterranean habitats, en F. di Castri, D.W. Goodall y R.L. Specht (eds.) Maquis and Chaparrals, Coll. Ecosystems of the world. Elsevier, Amsterdam, pp 361-385.

- (1985). Biogeografía y Ecología. Ed. Academia. León.

CALVO, J. F., e IBORRA, J. (1986). Estudio ecológico de la laguna de La Mata. Instituto de Estudios Juan Gil-Albert. Diputación Provincial de Alicante. Alicante.

CASTILLO, E., y RUIZ, F. (1977). Agroclimatología. Cuaderno INIA n. ${ }^{\circ} 7$. Ministerio de Agricultura, Pesca y Alimentación. Madrid.

CHEYLAN, G., y CHEYLAN, M. (1976). Biogéographie d'une montagne mediterranéenne: la Sainte Victoire (Bouches-du-Rhone). Alauda 44: 23-45.

ELÓSEGUI, J. (1985). Navarra. Atlas de aves nidificantes. Caja de Ahorros de Navarra.

GOLDEN SOFTWARE INC. (1985). Golden Graphics System. Colorado. U.S.A.

LEGENDRE, L. y LEGENDRE, P. (1979). Ecologie numérique. 2. La structure des données écologiques. Masson. Les presses de l'Université du Québec.

LÓPEZ, A., y ROSELLÓ, V. M. (1978). Geografía de la Provincia de Alicante. Excma. Diputación de Alicante. Alicante.

MACARTHUR, R. y WILSON, E.O. (1967). The theory of island biogeography. Princeton University Press. Princeton.

MARGALEF, R. (1974). Ecología. Ed. Omega. Barcelona.

MINISTERIO DE AGRICULTURA, PESCA Y ALIMENTACIÓN (1986). Mapa de cultivos y aprovechamientos de la provincia de Alicante. Servicio de Publicaciones Agrarias. Madrid.

MUNTANER, J., FERRER, X. y MARTÍNEZ-VILALTA, A. (1983). Atlas dels ocells nidificants de Catalunya i Andorra. Ed. Ketres. Barcelona.

POTTI, J. (1985). La sucesión de las comunidades de aves en los pinares repoblados de Pinus sylvestris del Macizo de Ayllón (Sistema Central). Ardeola, 32: 253-277.

SHARROCK, J. T. R. (1976). The atlas of breeding birds in Britain and Ireland. T. \& A. D. Poyser Ltd.

TELLERÍA, J. L. (1980). Datos preliminares sobre las características ecológicas y biogeográficas de las comunidades de aves del macizo de Ayllón. Bol. Est. Cent. Ecol., 18: 45-54.

- (1987). Biogeografía de la avifauna nidificante en España central. Ardeola, 34: 145-166.

THOMAS, D. (1979). Tasmanian Bird Atlas. University of Tasmania. Hobart.

VOOUS, K.H. (1960). Atlas of European Birds. Nelson. Amsterdam. 


\section{APÉNDICE I}

Distribución de las 151 especies en la provincia de Alicante. Los tres tipos de signo determinan las siguientes categorías. Nidificación segura

Nidificación probable

- Nidificación posible

El primer mapa muestra la segregación de la provincia según los UIM.

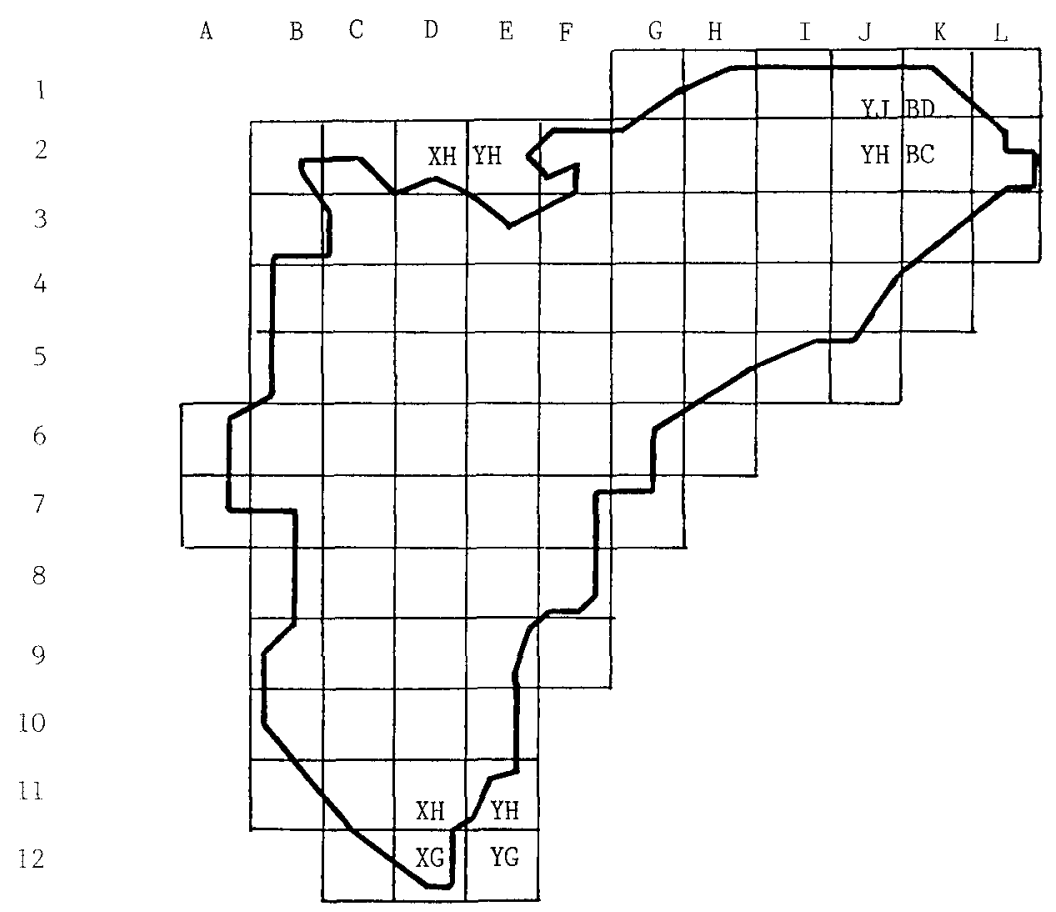

Figura 1.- 




Zampullín chico

(Tachybaptus ruficollis)



Zampullín cuellinegro

(Podiceps nigricollis)



Martinete (Nycticorax nycticorax)



Somormujo Lavanco (Podiceps cristatus)



Avetorillo (Ixobrichus minutus)



Garcilla cangrejera (Ardeola ralloides) 




Garcilla bueyera (Bubulcus ibis)



Garza Imperial (Ardea purpurea)



Anade friso (Anas strepera)



Garceta común (Egretta garzetta)



Tarro blanco (Tadorna tadorna)



Anade real (Anas platyrhynchos) 




Pato cuchara (Anas clypeata)



Pato colorado (Netta rufina)



Águila culebrera (Circaetus gallicus)



Cerceta pardilla

(Marmaronetta angustirrostris)



Porrón común (Aythya ferina)



Aguilucho Lagunero (Circus aeruginosus) 




Aguilucho cenizo (Circus pygargus)

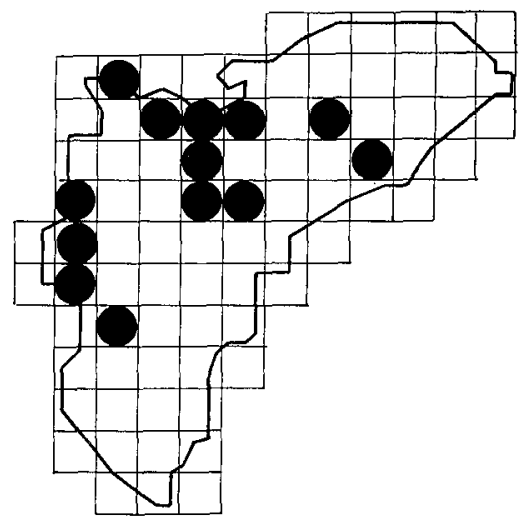

Gavilán (Accipiter nisus)



Águila real (Aquila chrysaetos)



Azor (Accipiter gentilis)



Ratonero común (Buteo buteo)

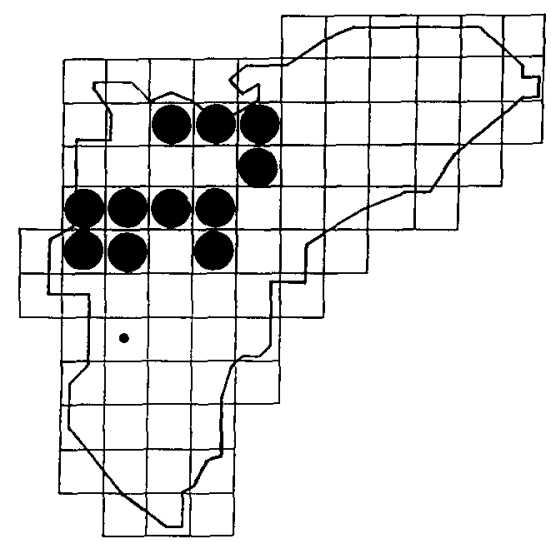

Águila calzada (Hieraetus pennatus) 


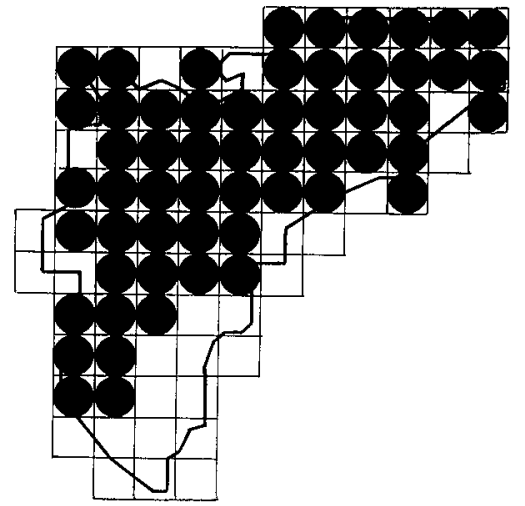

Águila perdicera (Hieraetus fasciatus)

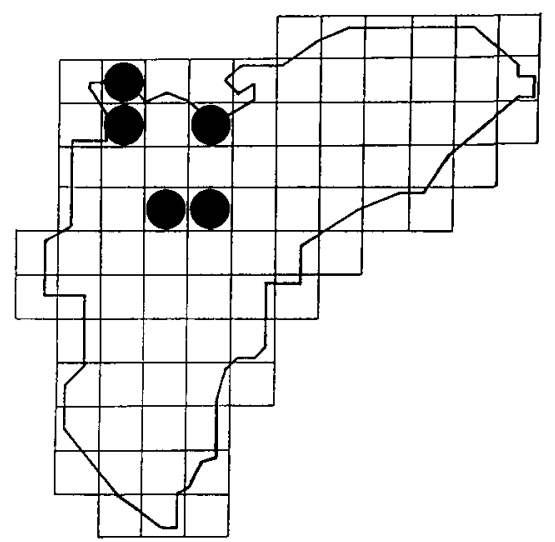

Alcotán (Falco subbuteo)



Perdiz común (Alectoris rufa)

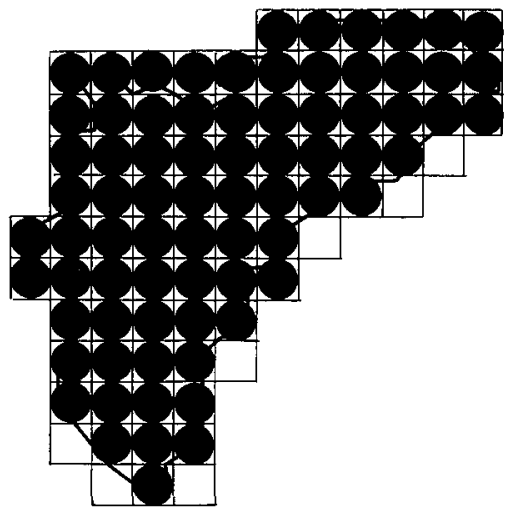

Cernícalo común (Falco tinnunculas)



Halcón común (Falco peregrinus)



Codorniz (Coturnix coturnix) 




Rascón (Rallus aquaticus)



Polla de agua (Gallinula chloropus)



Sisón (Tetrax tetrax)



Polluela chica (Porzana pusilla)



Focha (Fulica atra)



Cigüeñuela (Himantopus himantopus) 




Avoceta (Recurvirostra avosetta)

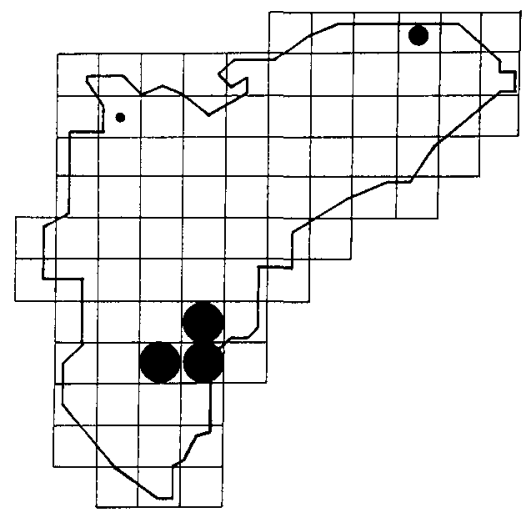

Canastera (Glareola pratincola)



Chorlitejo patinegro

(Charadrius alexandrinus)



Alcaraván (Burhinus oedicnemus)



Chorlitejo chico (Charadrius dubius)



Avefría (Vanellus vanellus) 


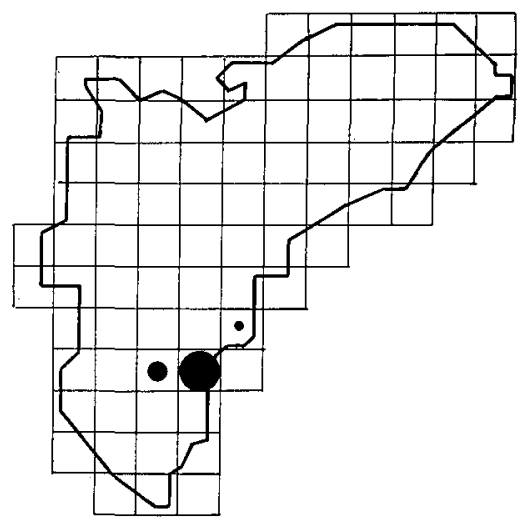

Archibebe común (Tringa totanus)

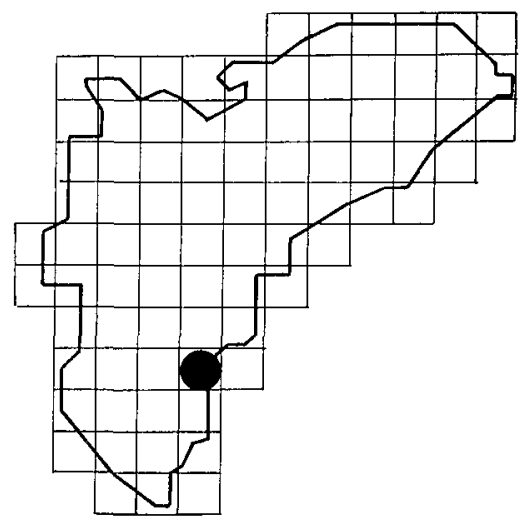

Gaviota reidora (Larus ridibundus)



Pagaza piconegra

(Gelochelidon nilotica)

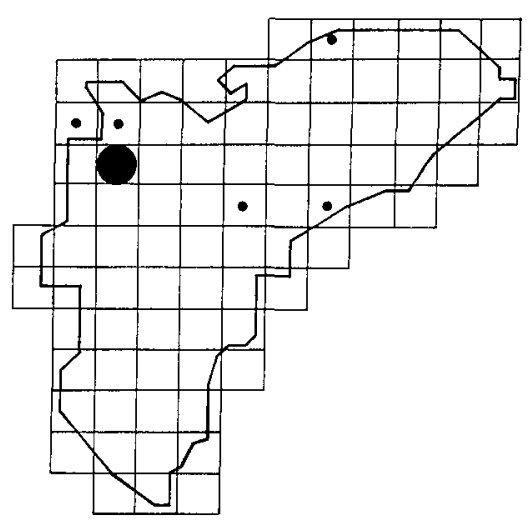

Andarríos chico (Actitis hypoleucos)

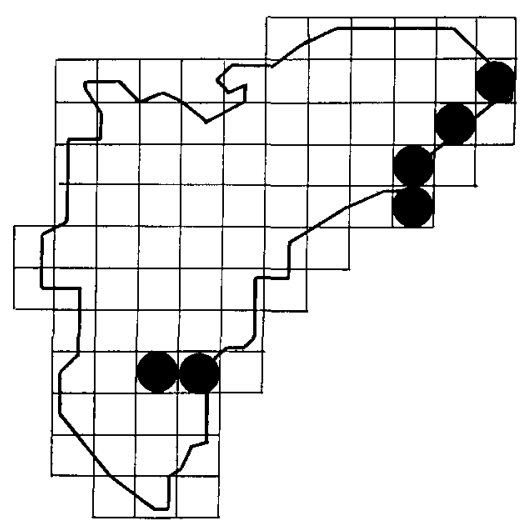

Gaviota argéntea (Larus argentatus)



Charrán común (Sterna hirundo) 


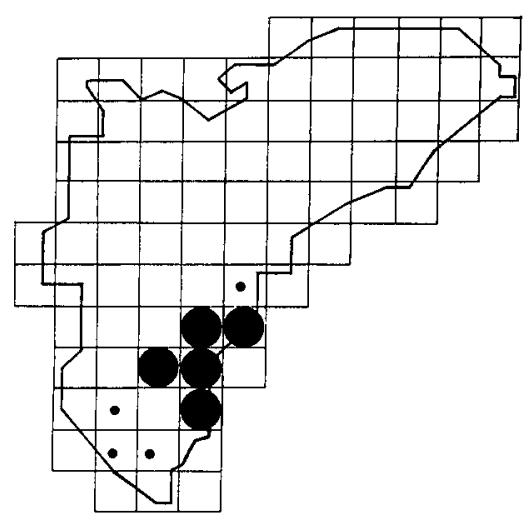

Charrancito (Sterna albifrons)

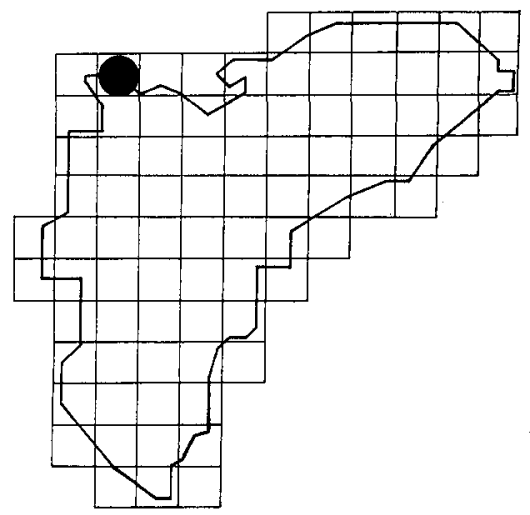

Ortega (Pterocles orientalis)

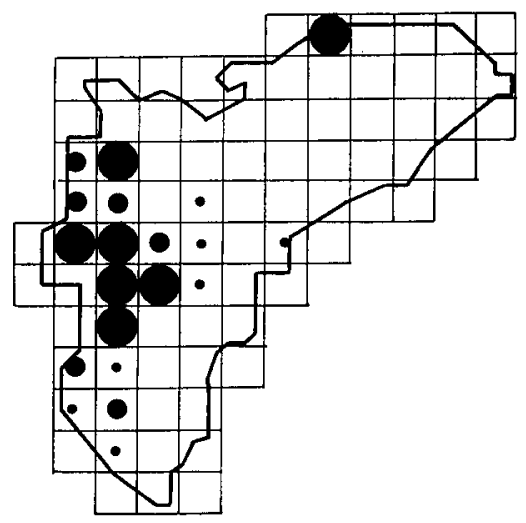

Paloma zurita (Columba oenas)

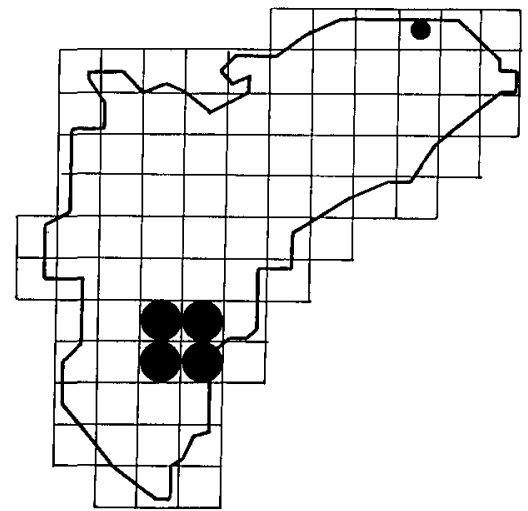

Fumarel cariblanco (Chlidonias hybrida)

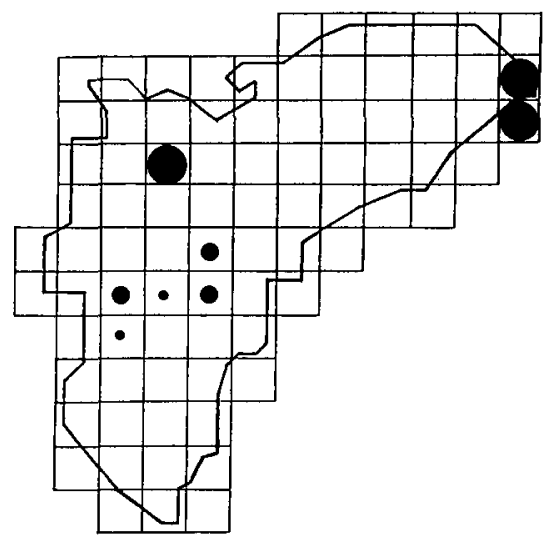

Paloma bravía (Columba livia)

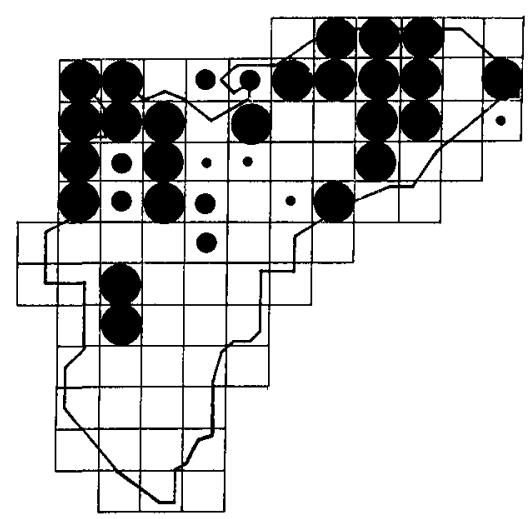

Paloma torcaz (Columba palumbus) 


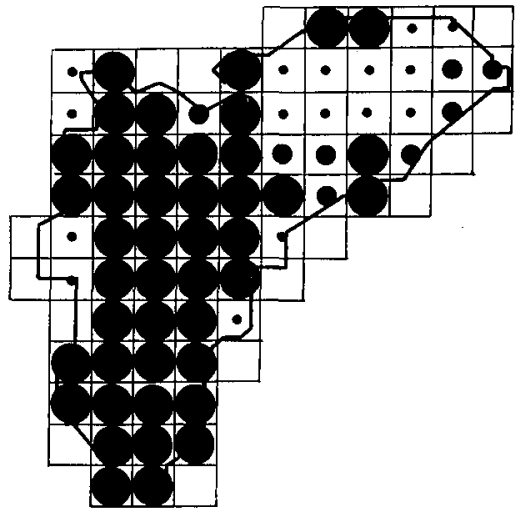

Tórtola (Streptopelia turtur)

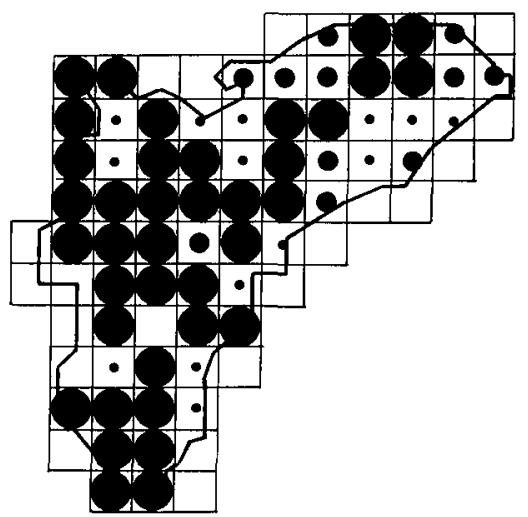

Cuco (Cuculus canorus)



Autillo (Otus scops)

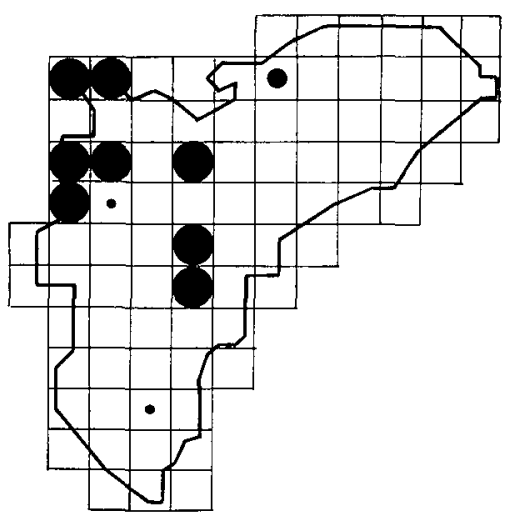

Críalo (Clamator glandarius)

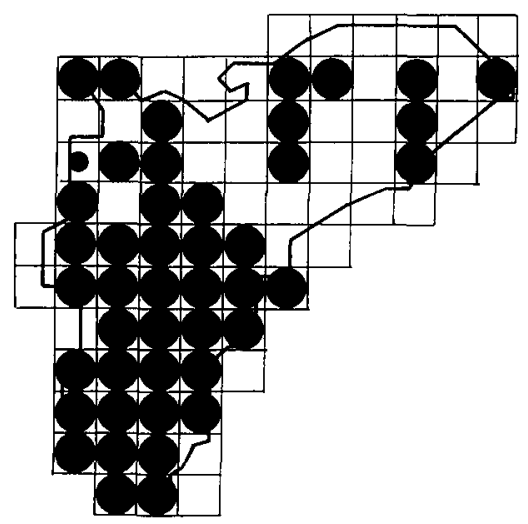

Lechuza (Tyto alba)

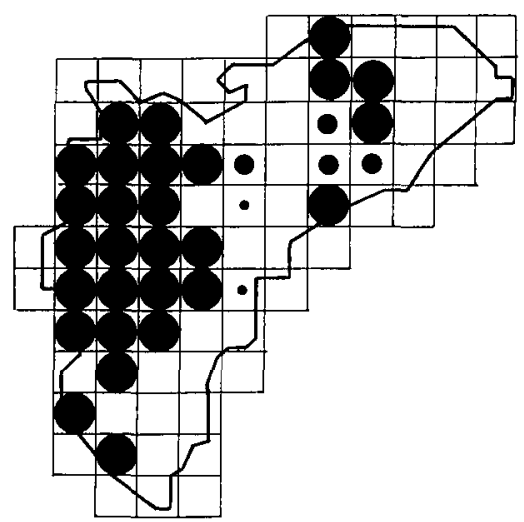

Buho real (Bubo bubo) 


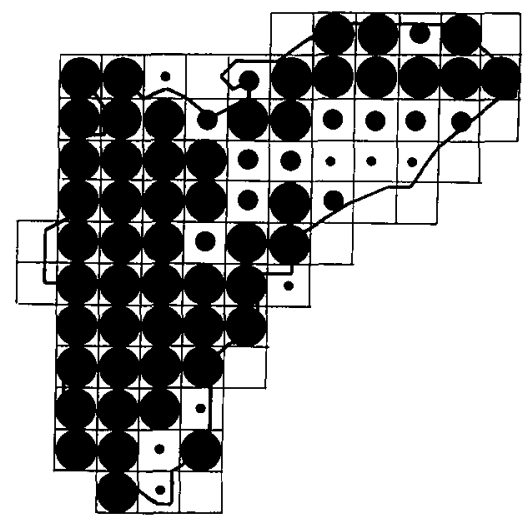

Mochuelo (Athene noctua)

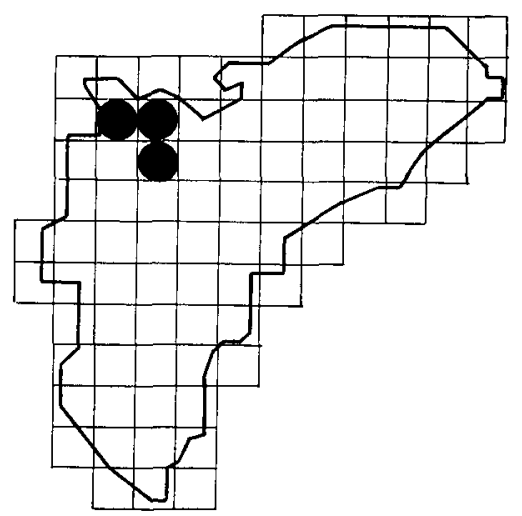

Buho chico (Asio otus)

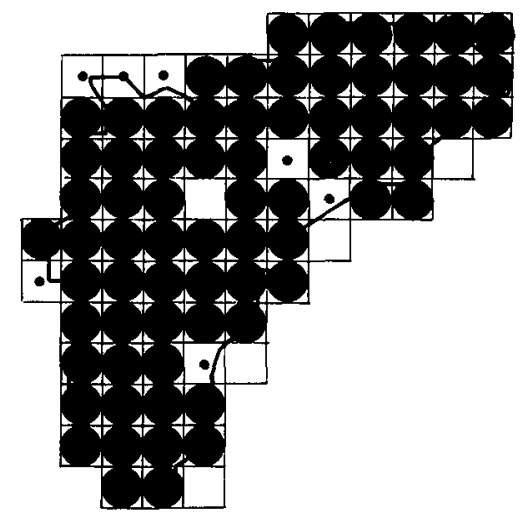

Vencejo común (Apus apus)

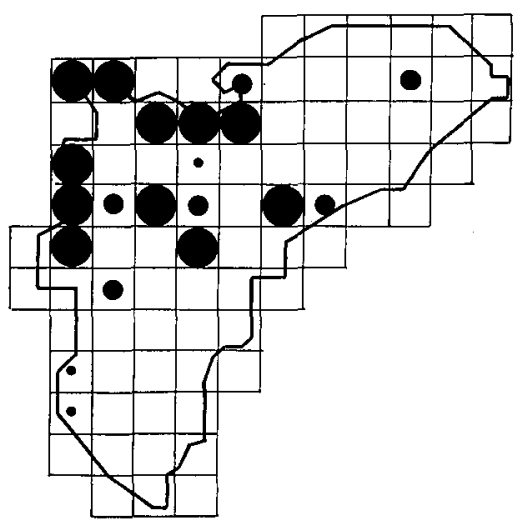

Cárabo (Strix aluco)

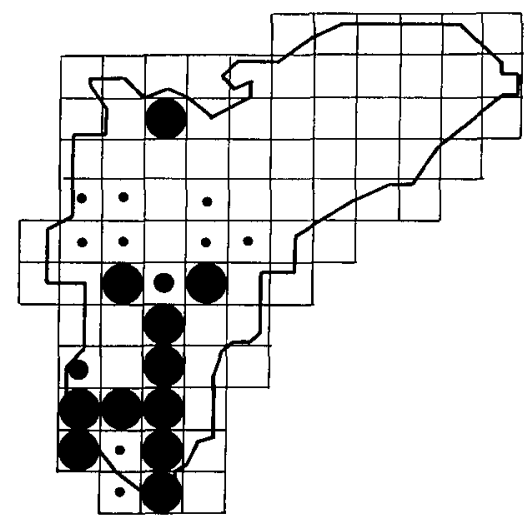

Chotacabras pardo

(Caprimulgus ruficollis)

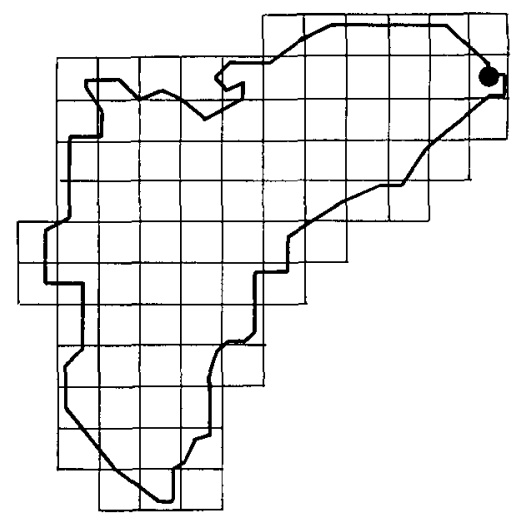

Vencejo pálido (Apus pallidus) 


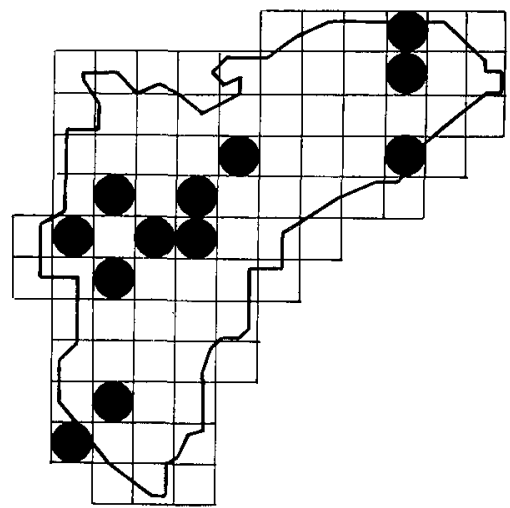

Vencejo real (Apus melba)

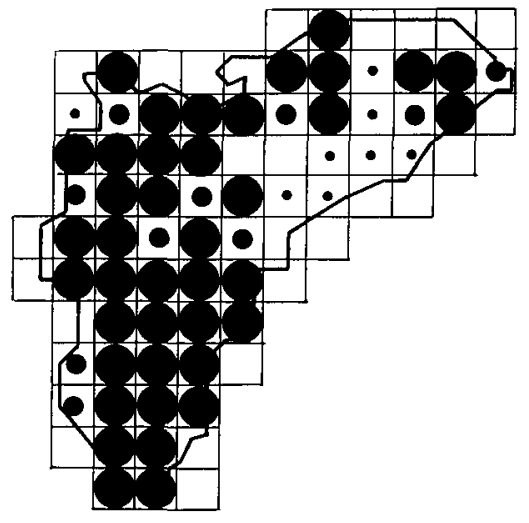

Abejaruco (Merops apiaster)

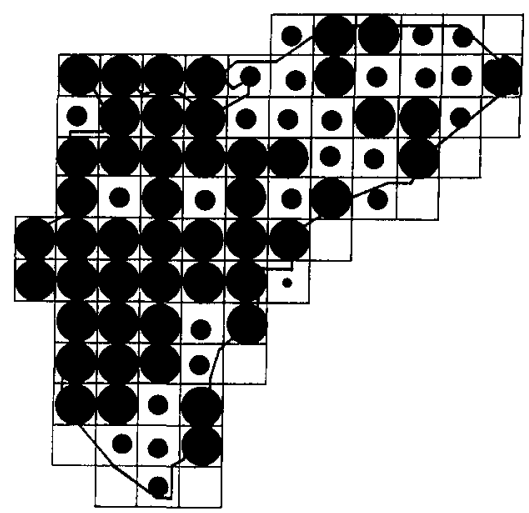

Abubilla (Upupa epops)

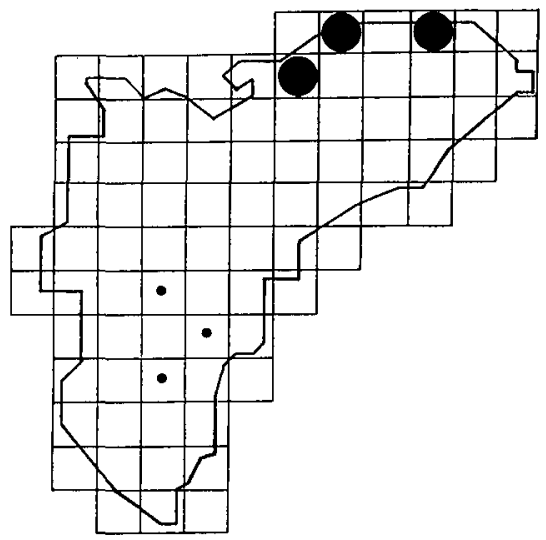

Martín pescador (Alcedo atthis)

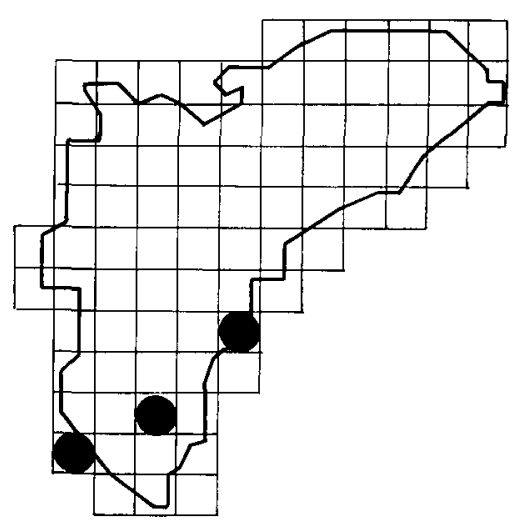

Carraca (Coracias garrulus)

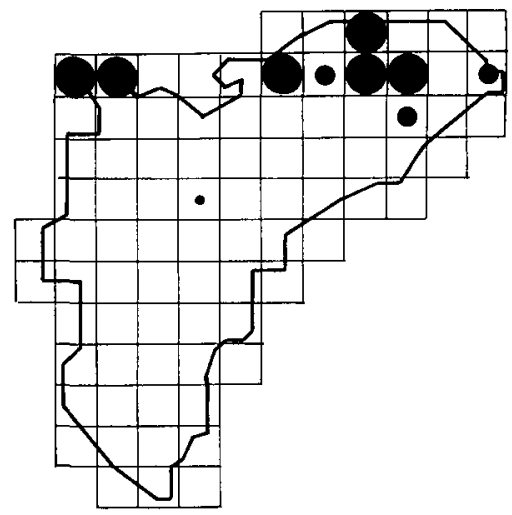

Torcecuello (Jynx torquilla) 


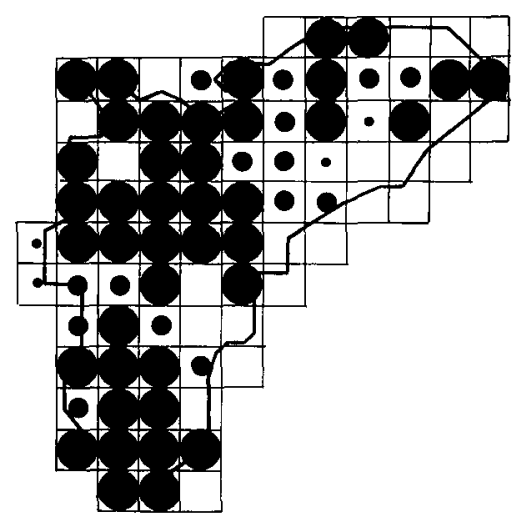

Pito real (Picus viridis)

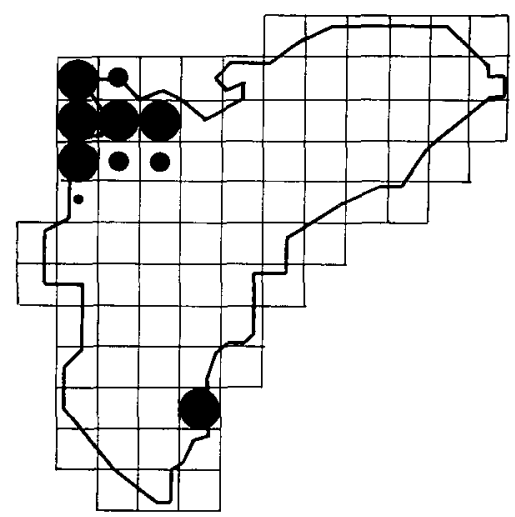

Calandria (Melanocorhypha calandra)

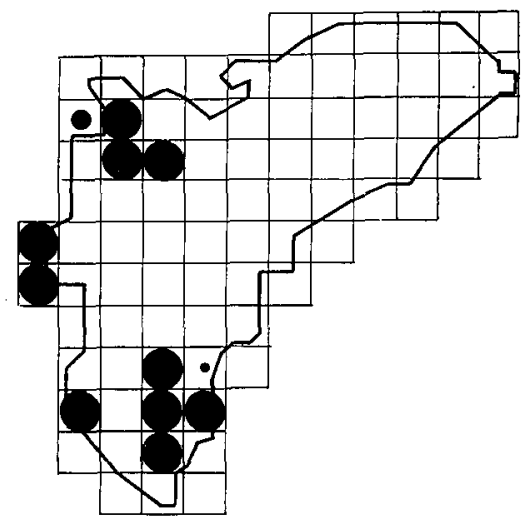

Terrera marismeńa

(Calandrella rufescens)

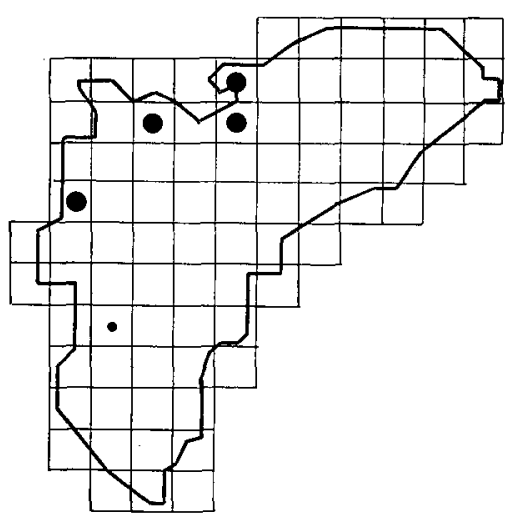

Pico picapinos (Dendrocopos major)

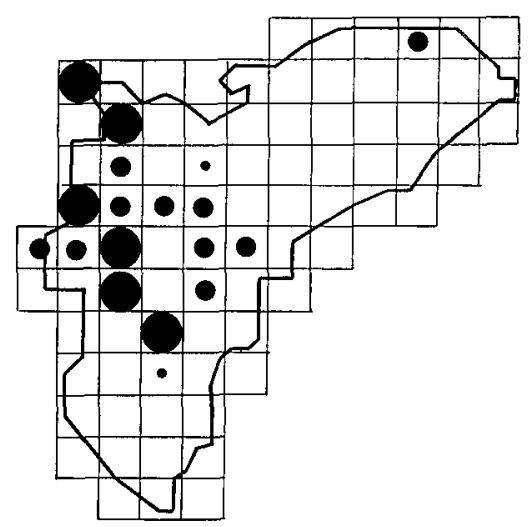

Terrera común (Calandrella cinerea)

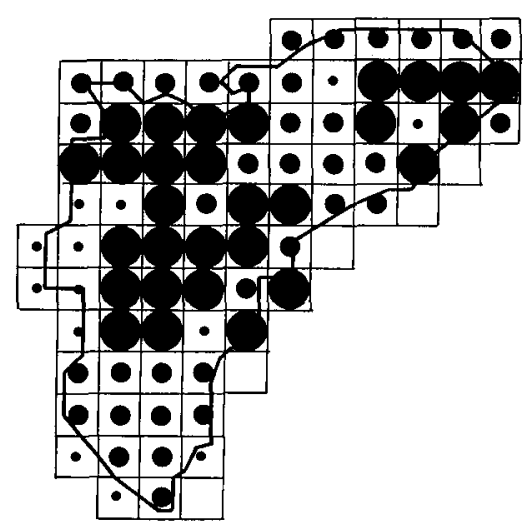

Cogujada común (Galerida cristata) 


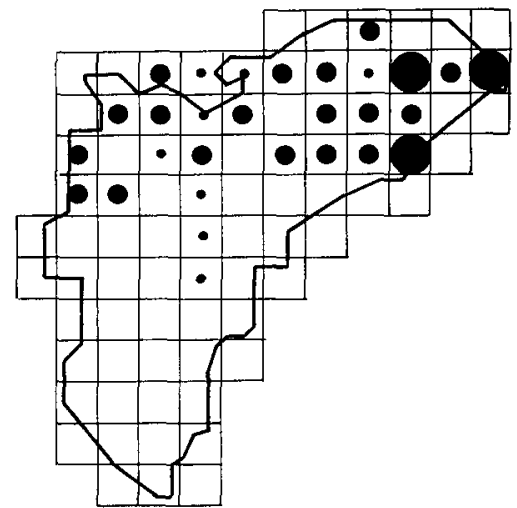

Cogujada montesina (Galerida teklae)

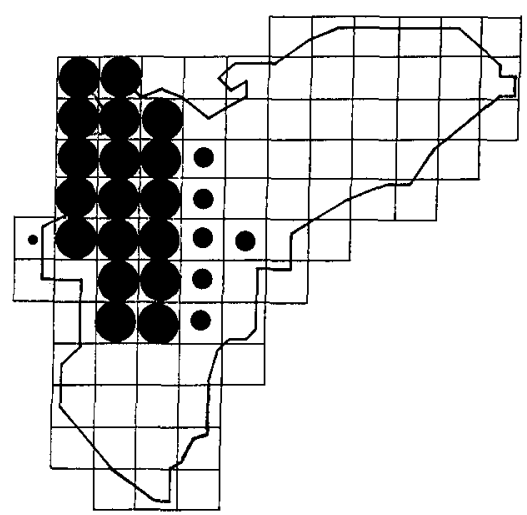

Alondra (Alauda arvensis)

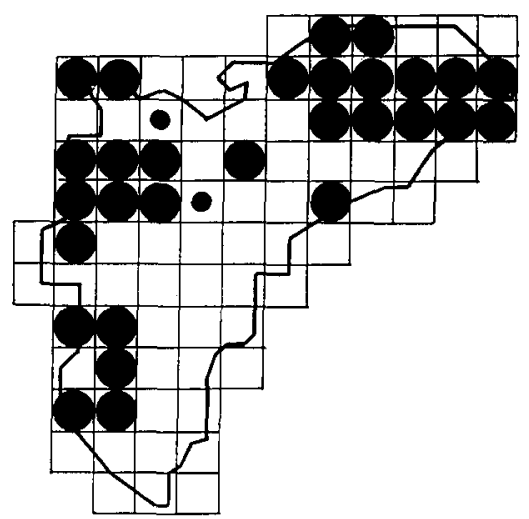

Avión roquero (Ptyonoprogne rupestris)

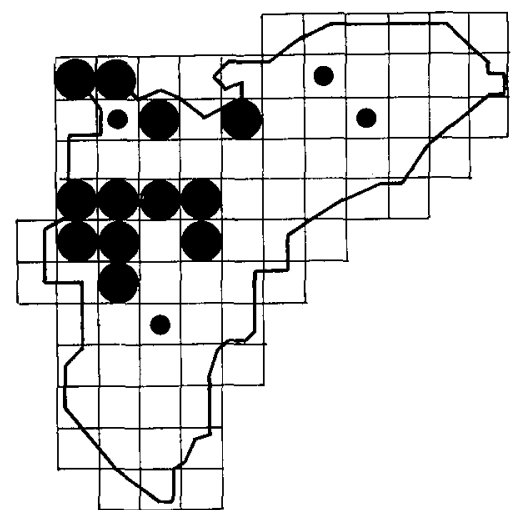

Totovía (Lullula arborea)

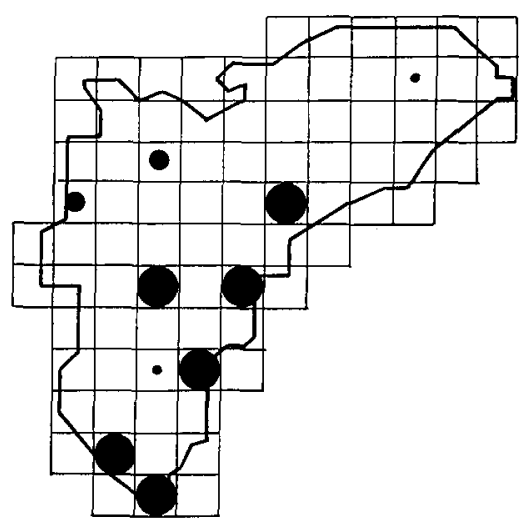

Avión zapador (Riparia riparia)

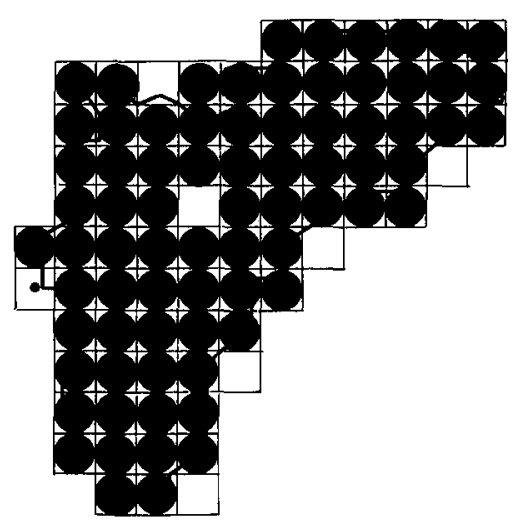

Golondrina común (Hirundo rustica) 


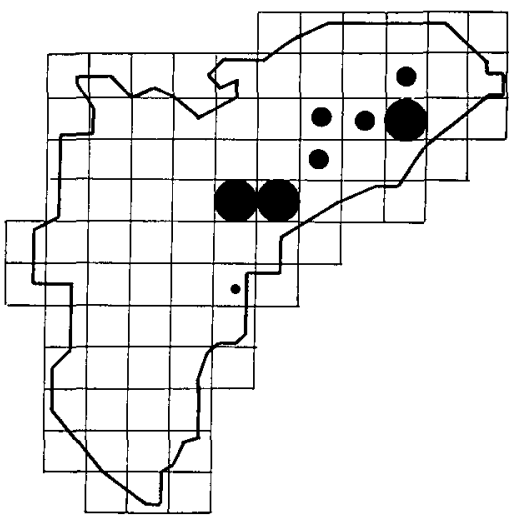

Golondrina daúrica (Hirundo daurica)

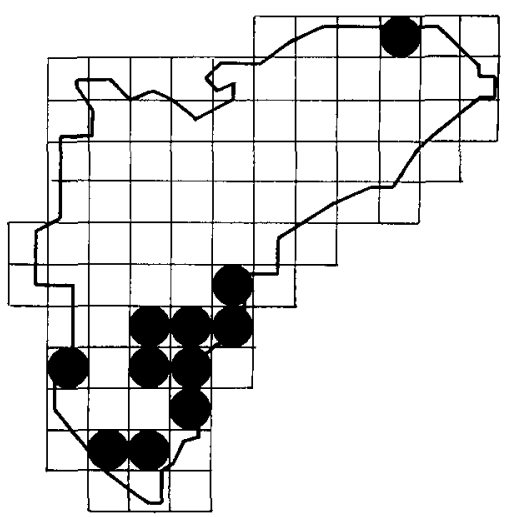

Lavandera boyera (Motacilla flava)

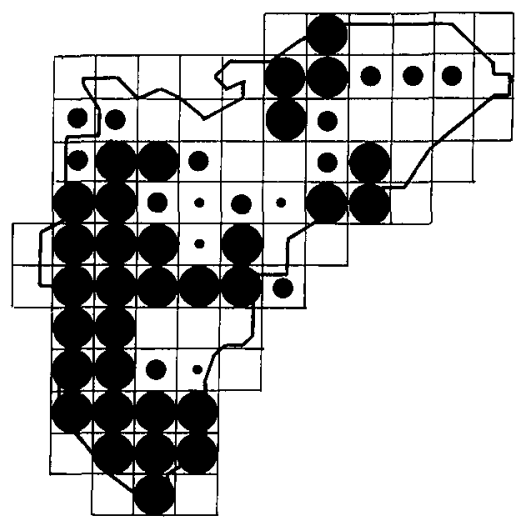

Lavandera blanca (Motacilla alba)

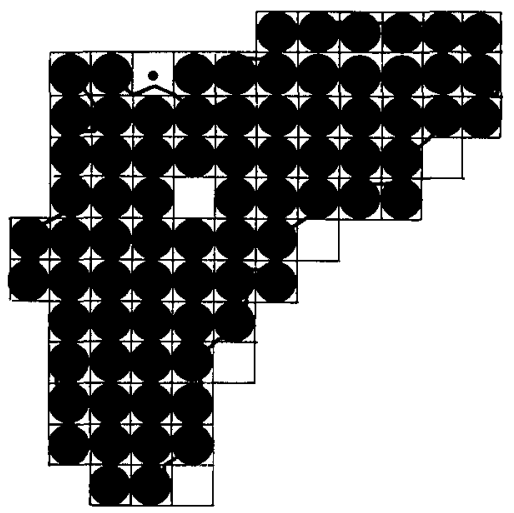

Avión común (Delichon urbica)

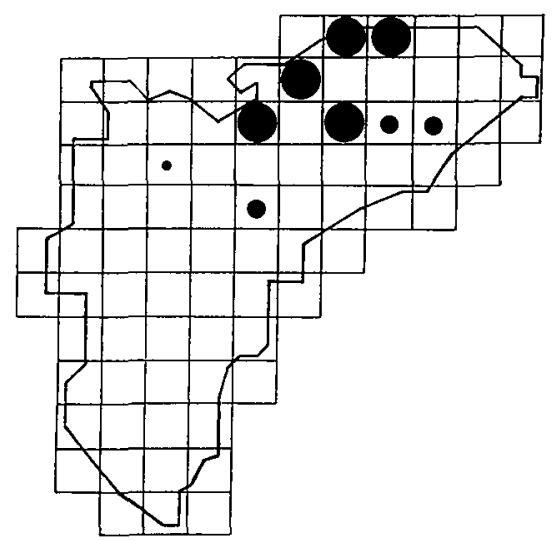

Lavandera cascadeña (Motacilla cinerea)

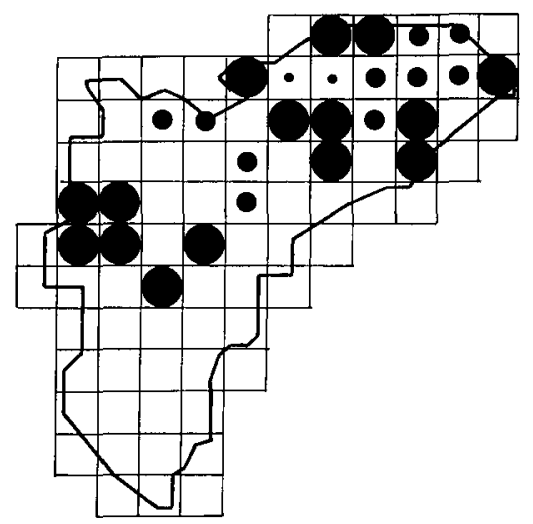

Chochin (Troglodytes troglodytes) 


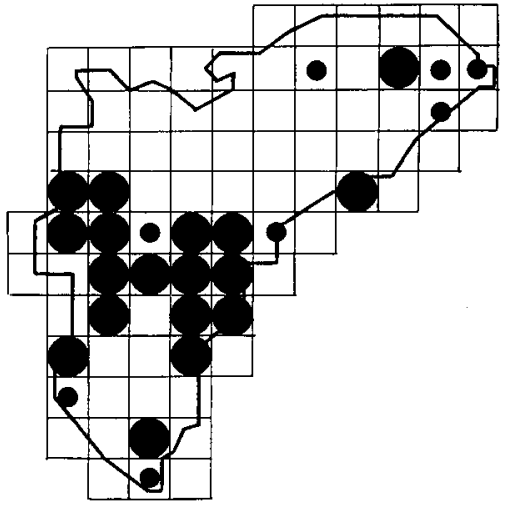

Alzacola (Cercotrichas galactotes)

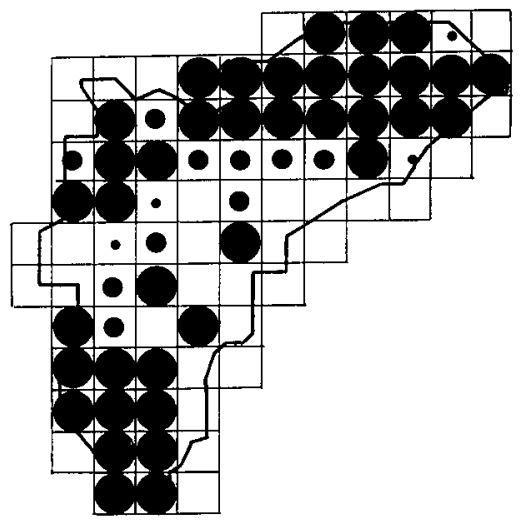

Ruiseñor común

(Luscinia megarhynchos)

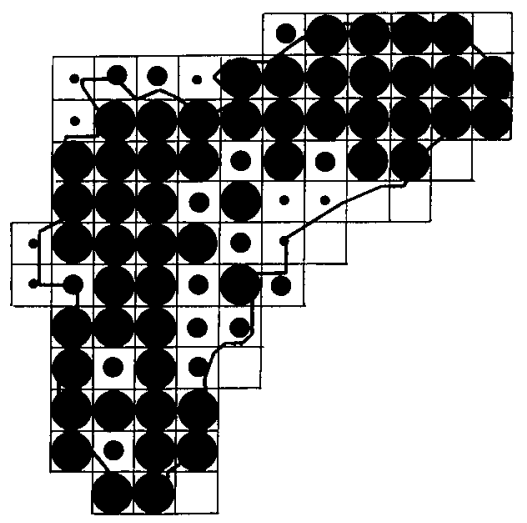

Tarabilla común (Saxicola torquata)

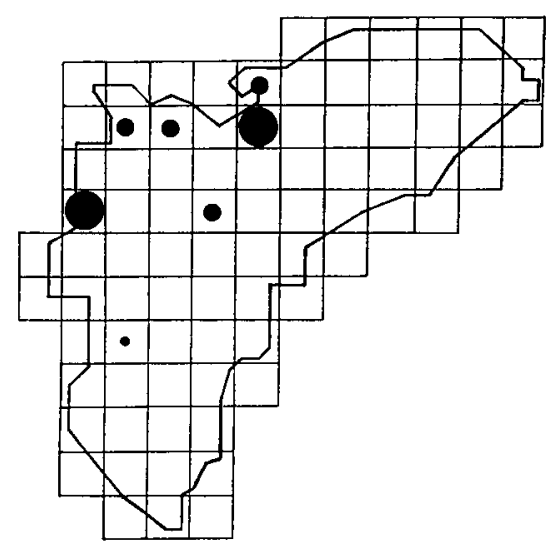

Petirrojo (Erithacus rubecula)

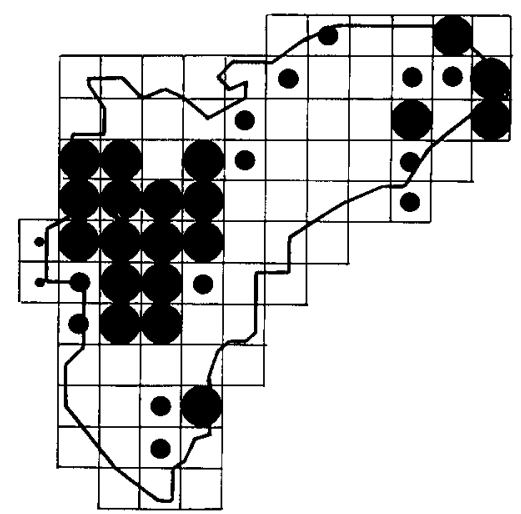

Colirrojo tizón (Phoenicurus ochruros)

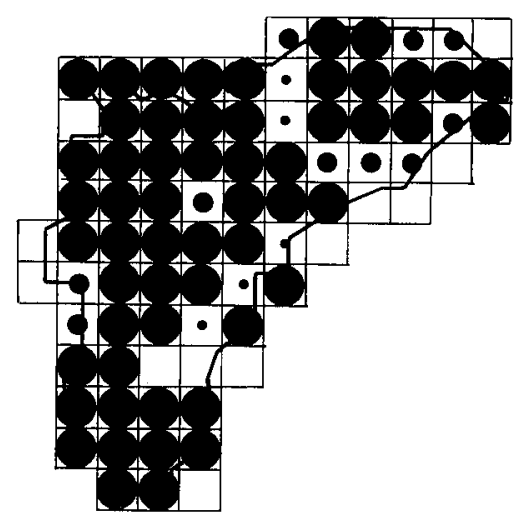

Collalba rubia (Oenanthe hispanica) 


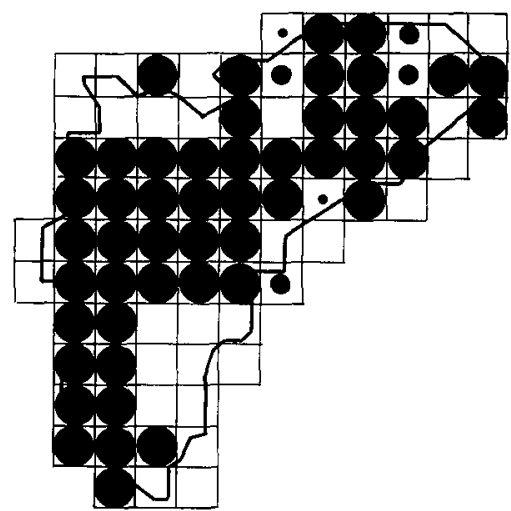

Collalba negra (Oenanthe leucura)

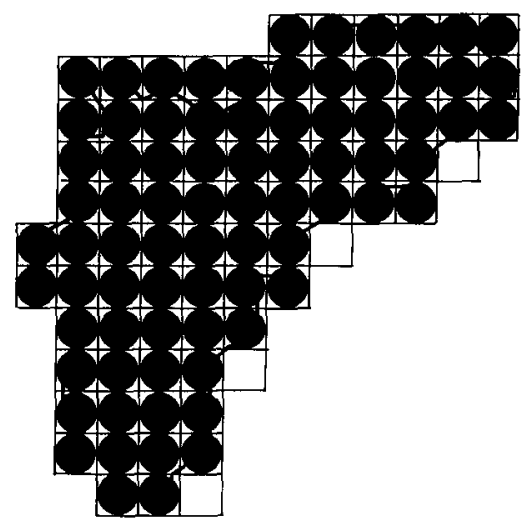

Mirlo común (Turdus merula)

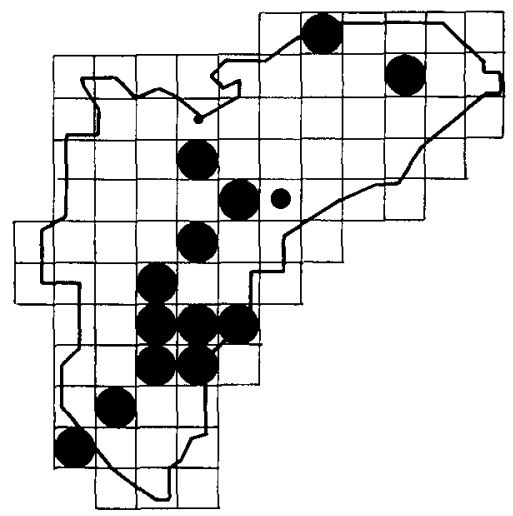

Ruiseñor bastardo (Cettia cetti)

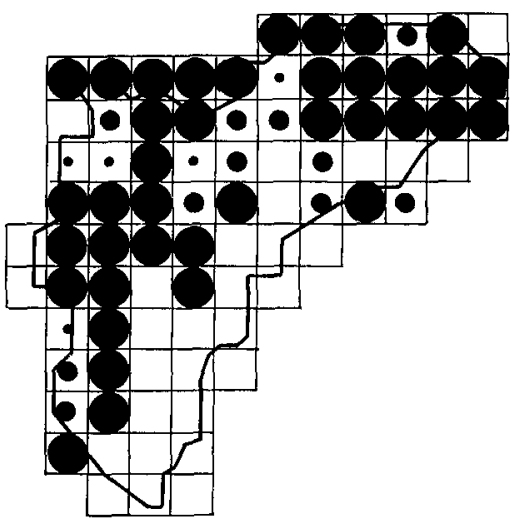

Roquero solitario (Monticola solitarius)

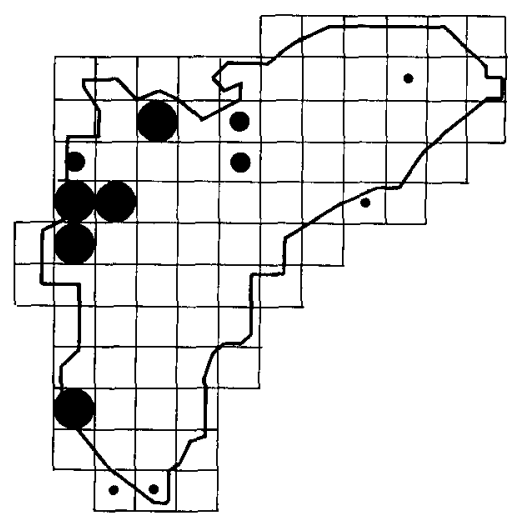

Zorzal charlo (Turdus viscivorus)

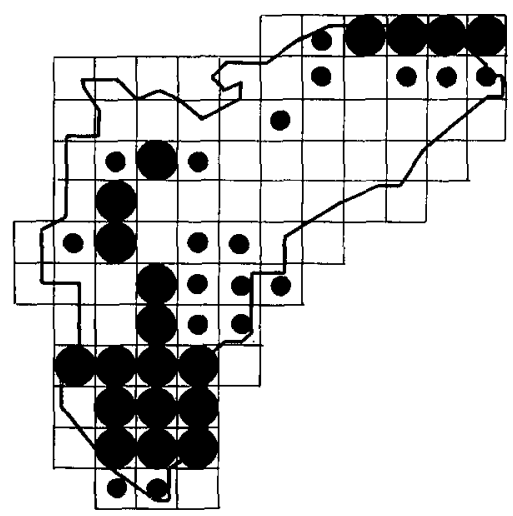

Buitrón (Cisticola juncidis) 


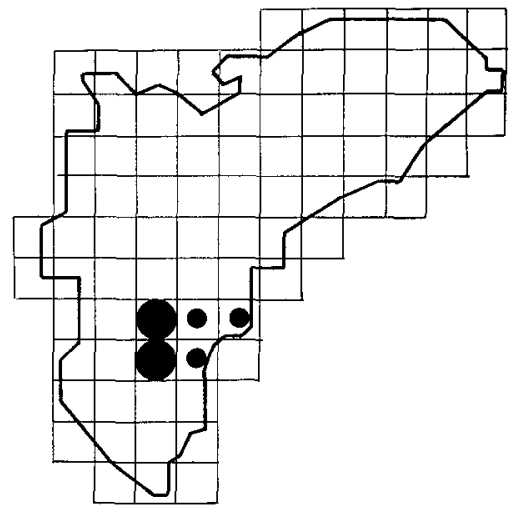

Buscarla unicolor (Locustella luscinioides)

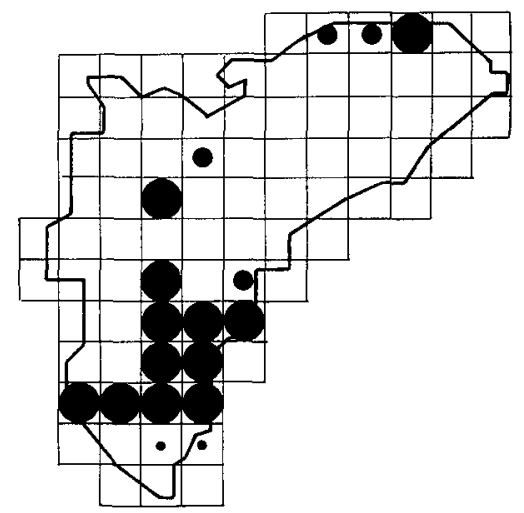

Carricero común

(Acrocephalus scirpaceus)

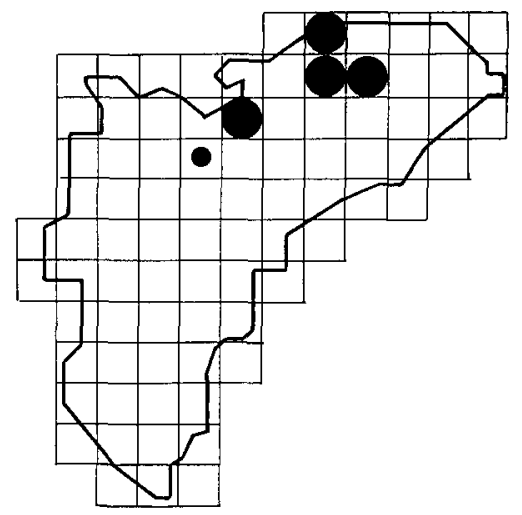

Zarcero pálido (Hippolais pallida)

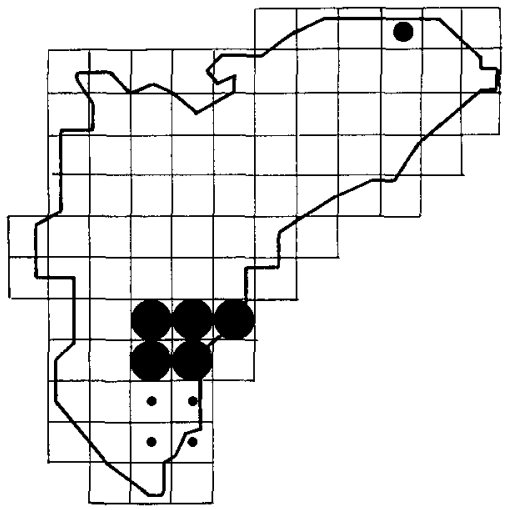

Carricerín real

(Acrocephalus melanopogon)

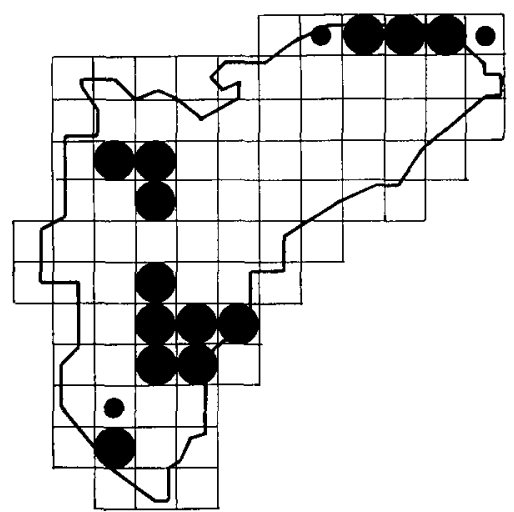

Carricero tordal

(Acrocephalus arundinaceus)

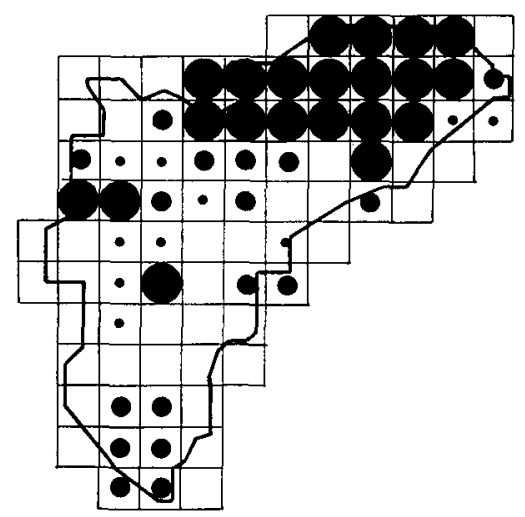

Zarcero común (Hippolais polyglotta) 


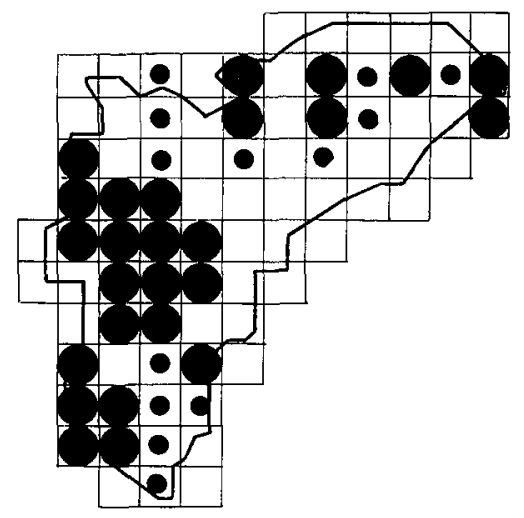

Curruca rabilarga (Sylvia undata)

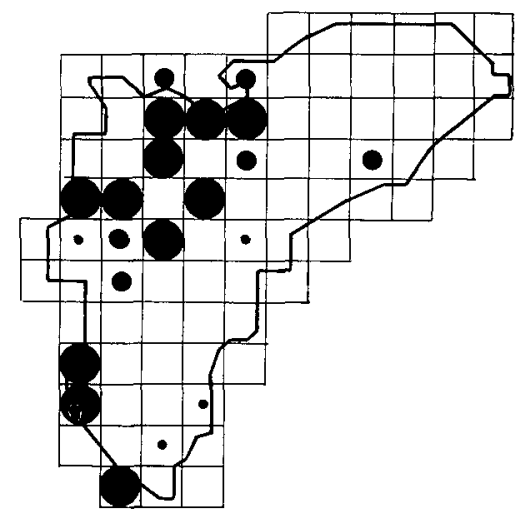

Curruca carrasqueña (Sylvia cantỉllans)

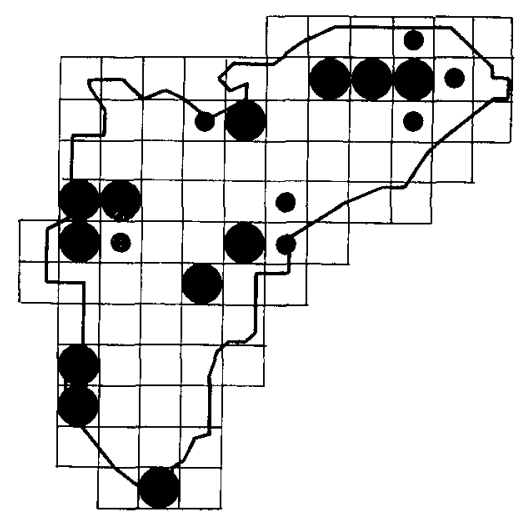

Curruca miriona (Sylvia hortensis)

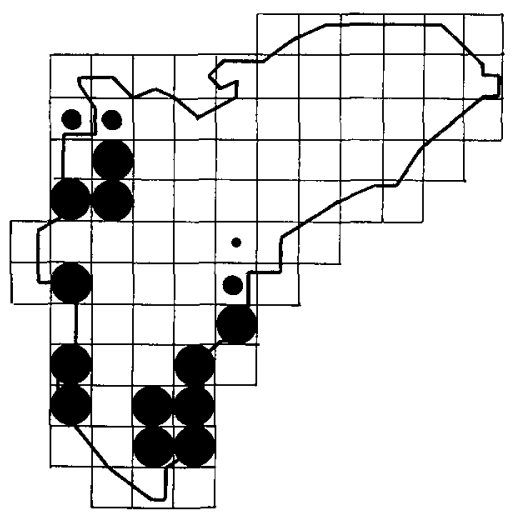

Curruca tomillera (Sy/via conspicillata)

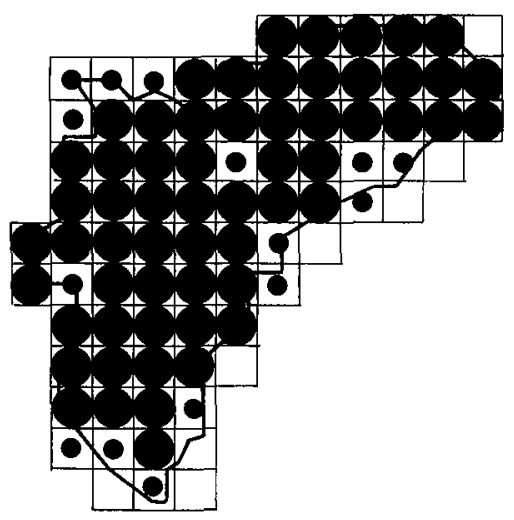

Curruca cabecinegra

(Sylvia melanocephala)

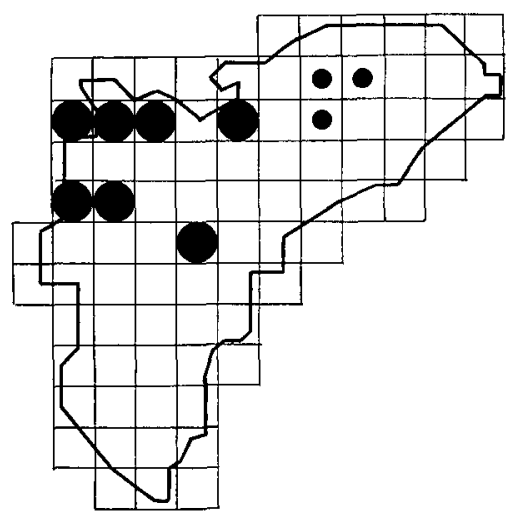

Curruca zarcera (Sylvia communis) 


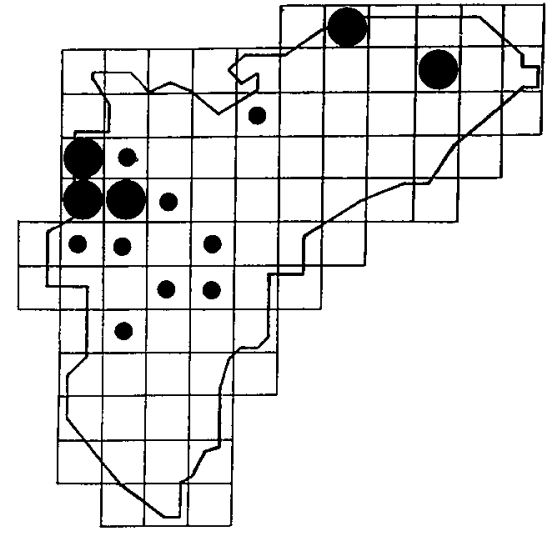

Curruca capirotada (Sylvia atricapilla)

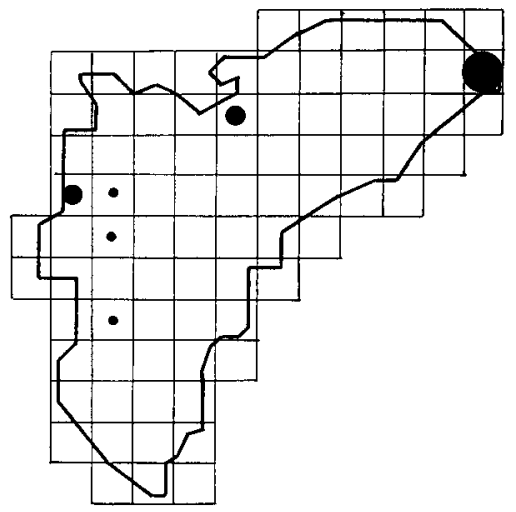

Reyezuelo listado (Regulus ignicapillus)

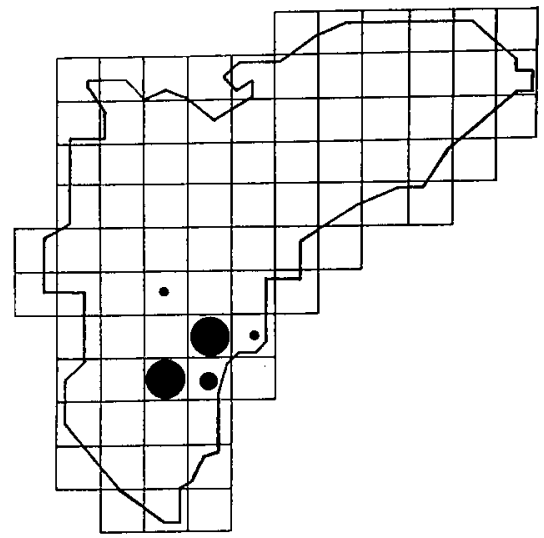

Bigotudo (Panurus biarmicus)

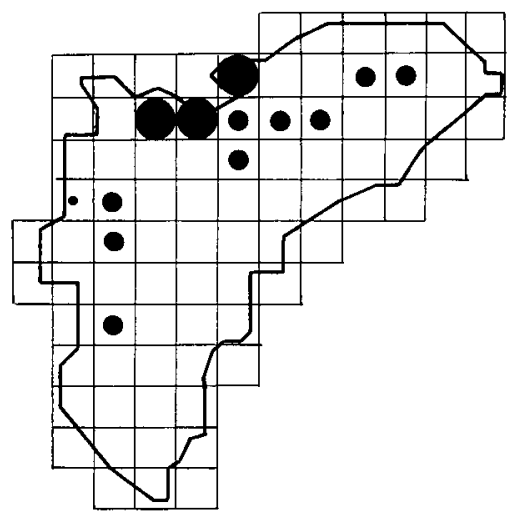

Mosquitero papialbo (Phylloscopus bonelli)

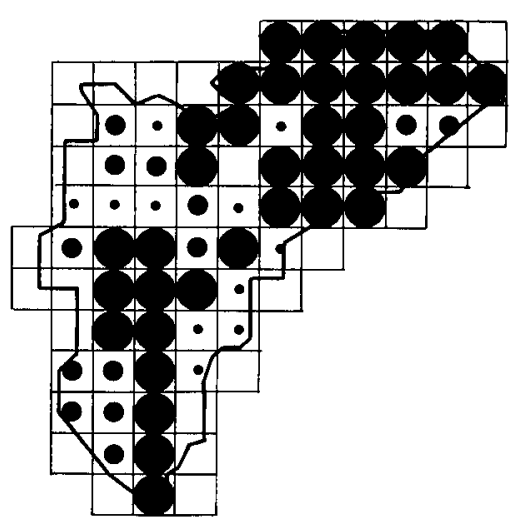

Papamoscas gris (Muscicapa striata)

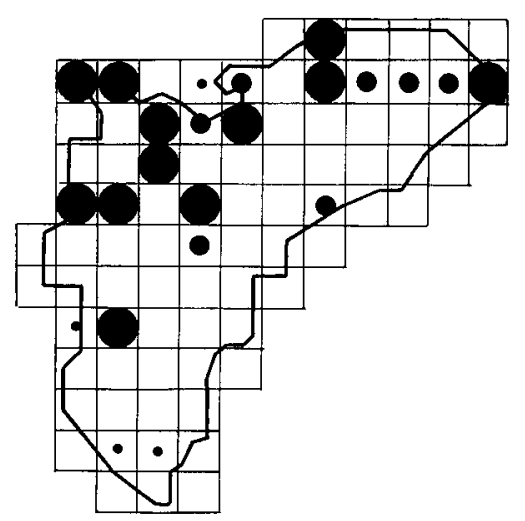

Mito (Aegithalos caudatus) 


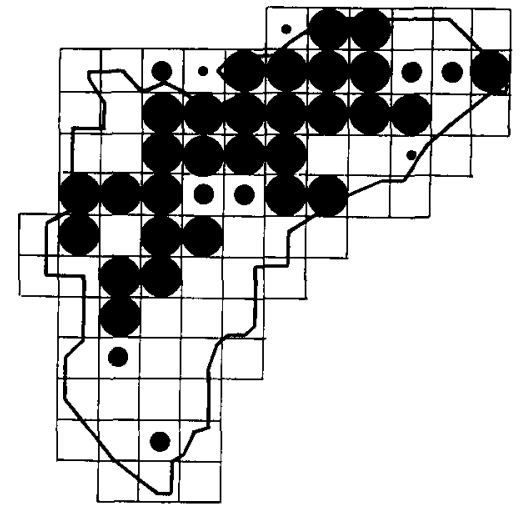

Herrerillo capuchino (Parus cristatus)

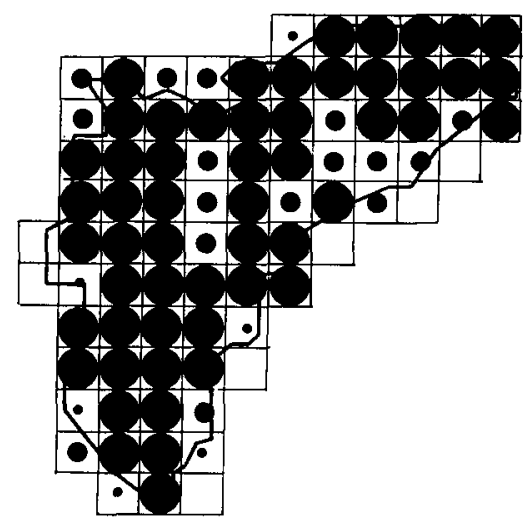

Carbonero común (Parus major)

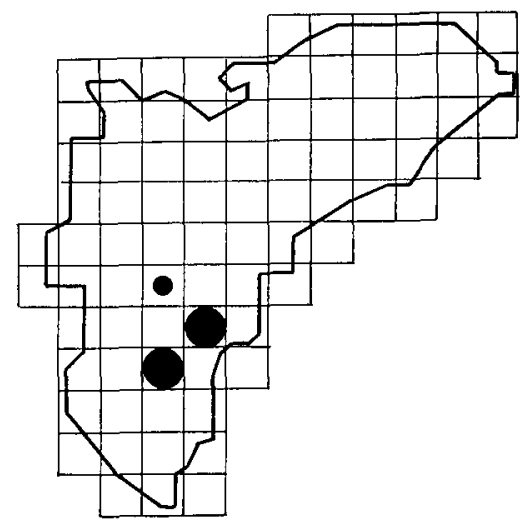

Pájaro moscón (Remiz pendulinus)

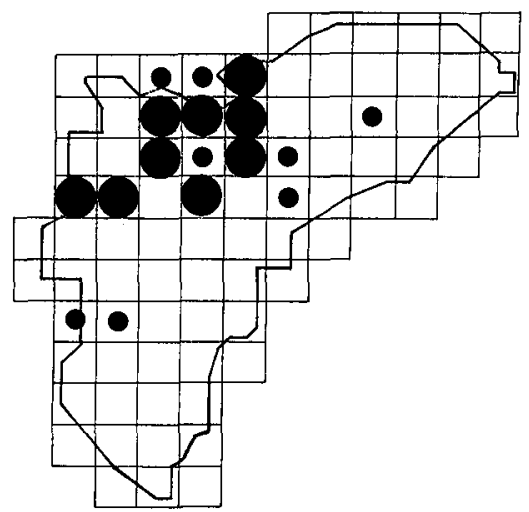

Carbonero garrapinos (Parus ater)

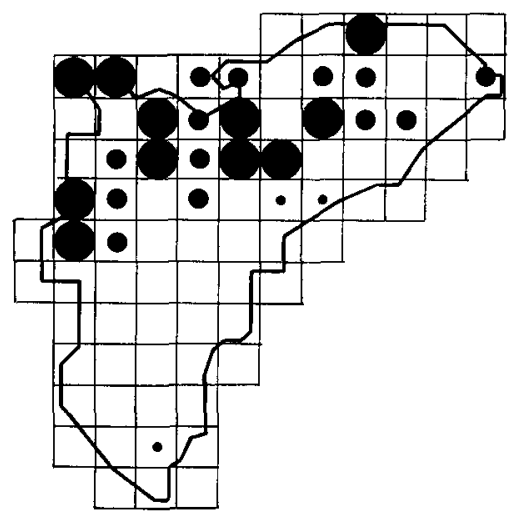

Agateador común

(Certhia brachydactyla)

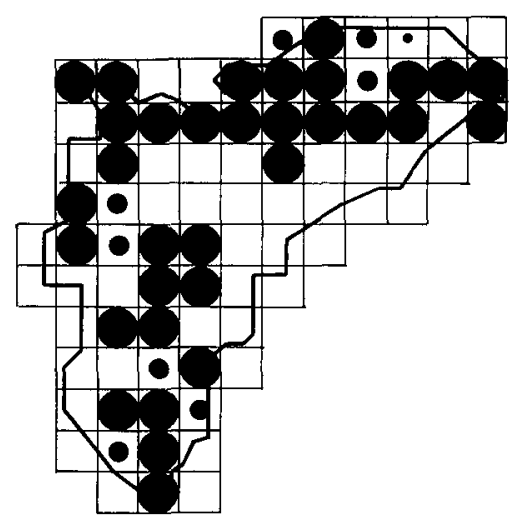

Oropéndola (Oriolus oriolus) 


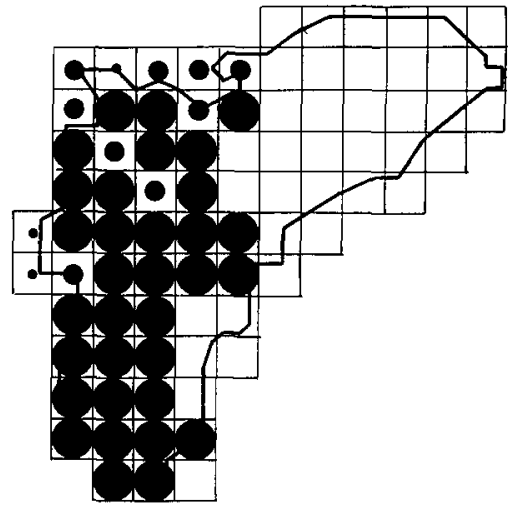

Alcaudón real (Lanius excubitor)

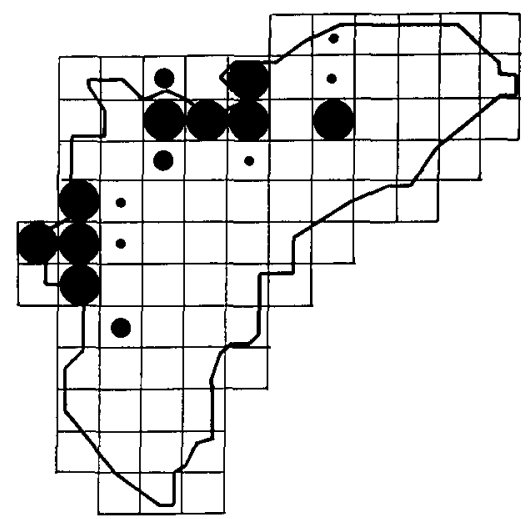

Arrendajo (Garrulus glandarius)

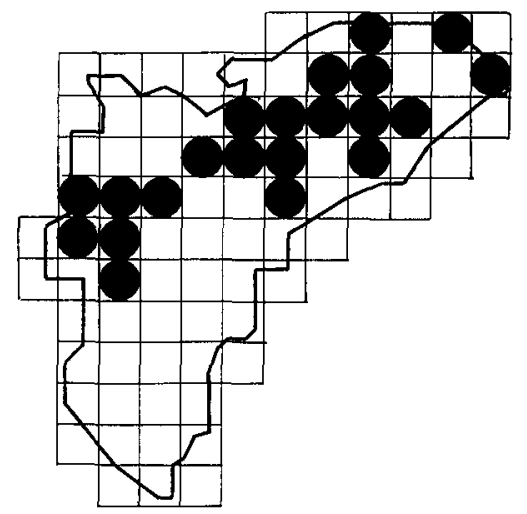

Chova piquirroja

(Pyrrhocorax pyrrhocorax)

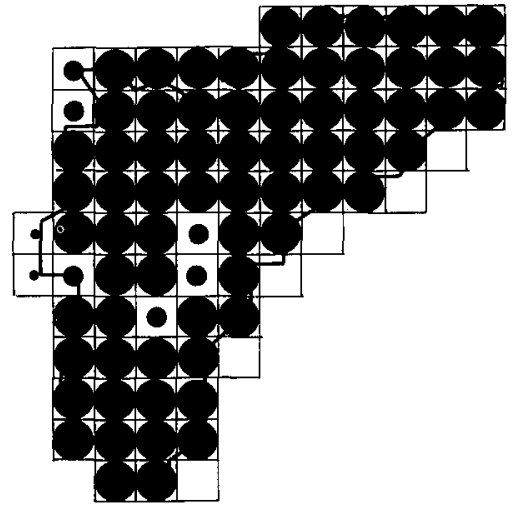

Alcaudón común (Lanius senator)

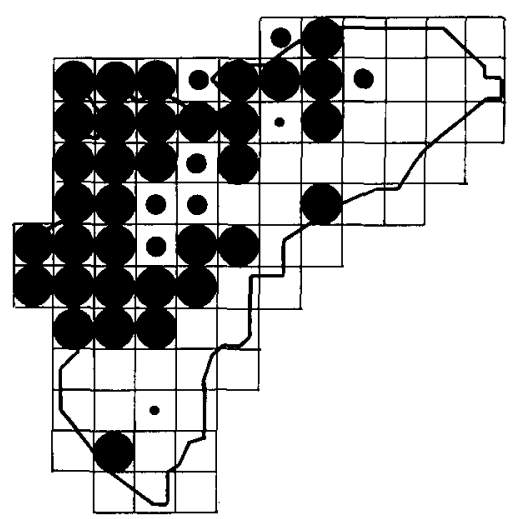

Urraca (Pica pica)

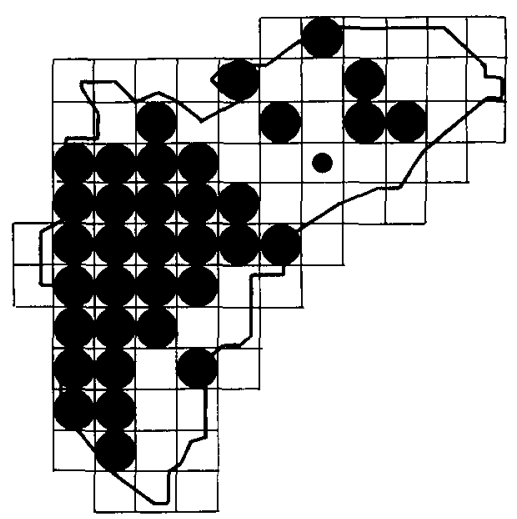

Grajilla (Corvus monedula) 


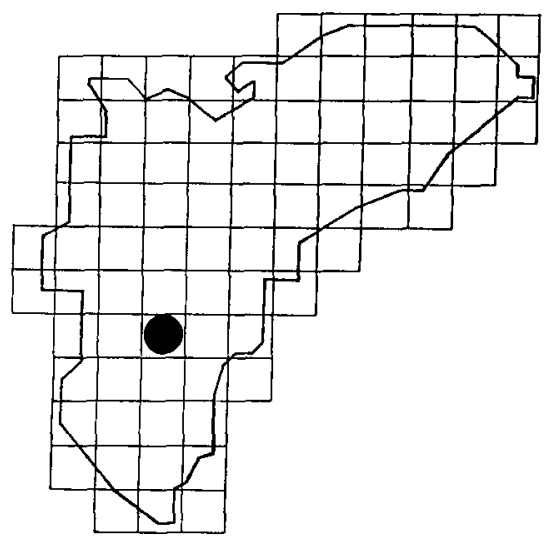

Corneja negta (Corvus corone)

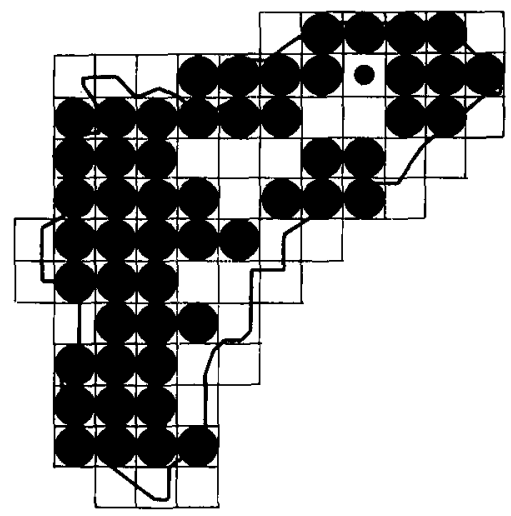

Estornino negro (Sturnus unicolor)

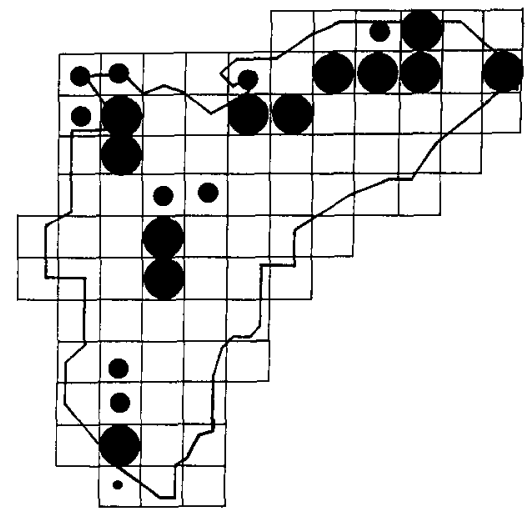

Gorrión molinero (Passer montanus)

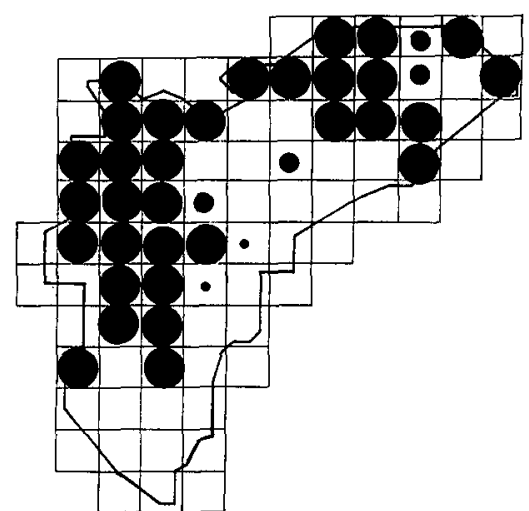

Cuervo (Corvus corax)

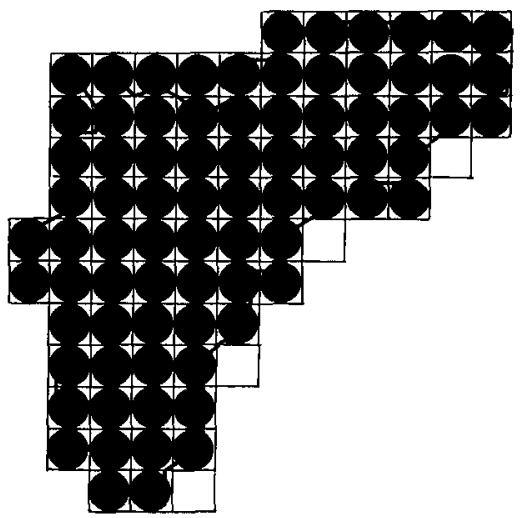

Gorrión común (Passer domesticus)

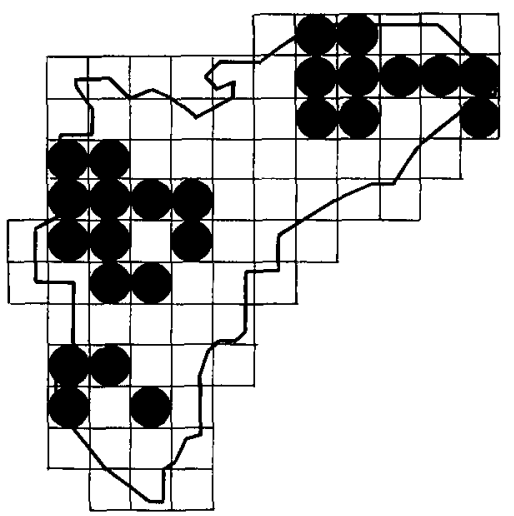

Gorrión chillón (Petronia petronia) 


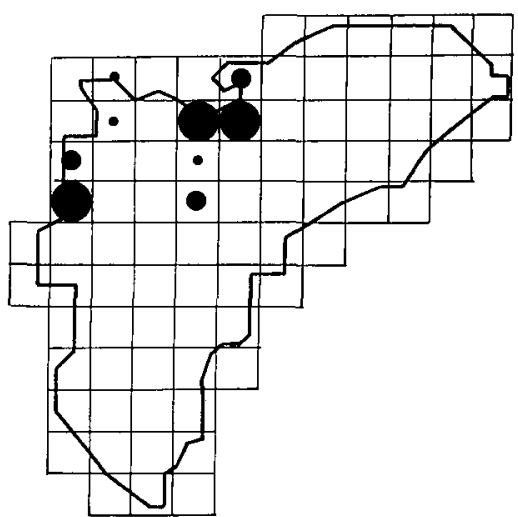

Pinzón común (Fringilla coelebs)

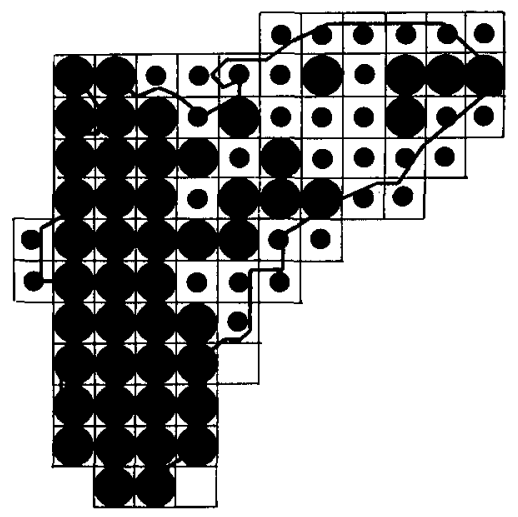

Verderón común (Carduelis chloris)

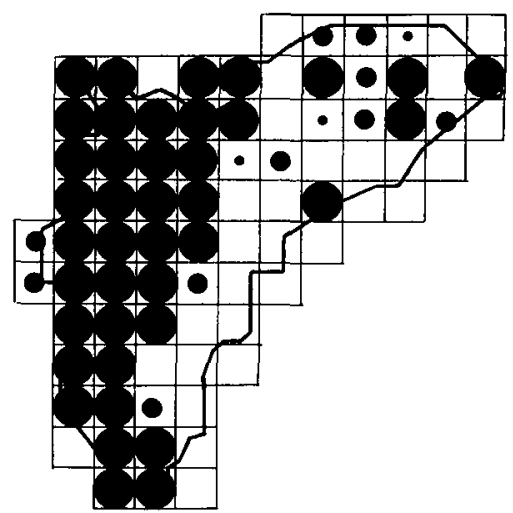

Pardillo común (Carduelis cannabina)

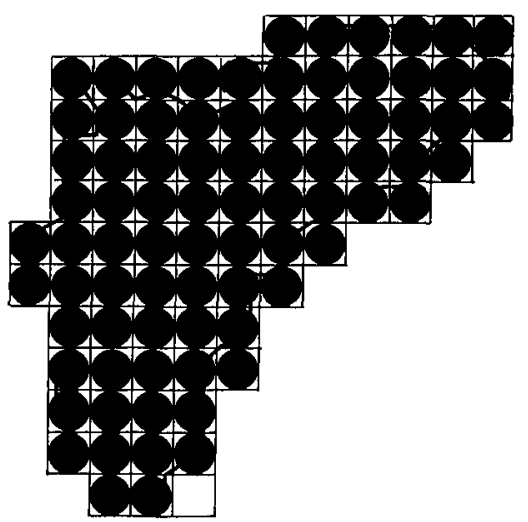

Verdecillo (Serinus serinus)

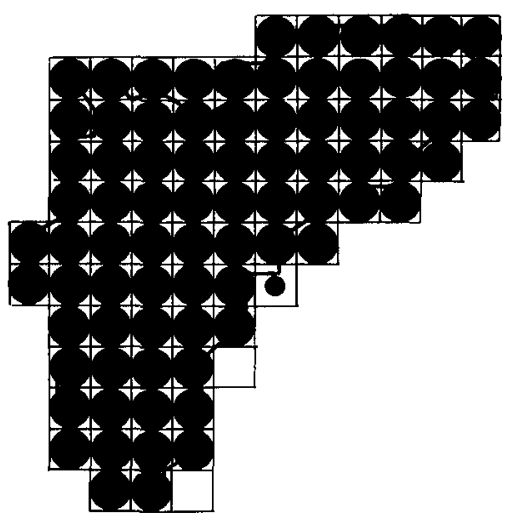

Jilguero (Carduelis carduelis)

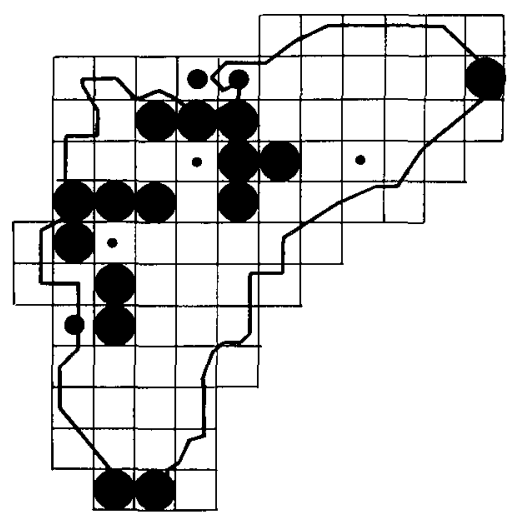

Piquituerto común (Loxia curvirostra) 


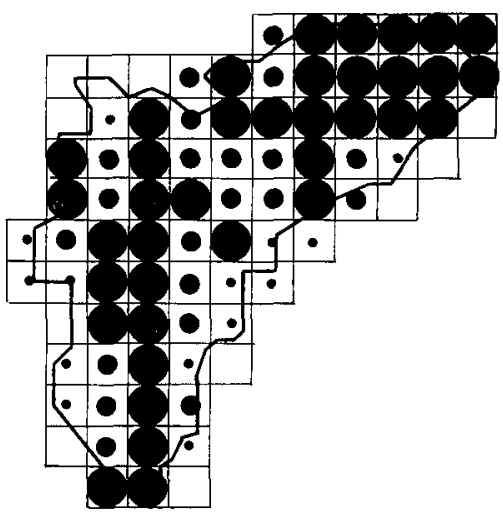

Escribano soteño (Emberiza cirlus)

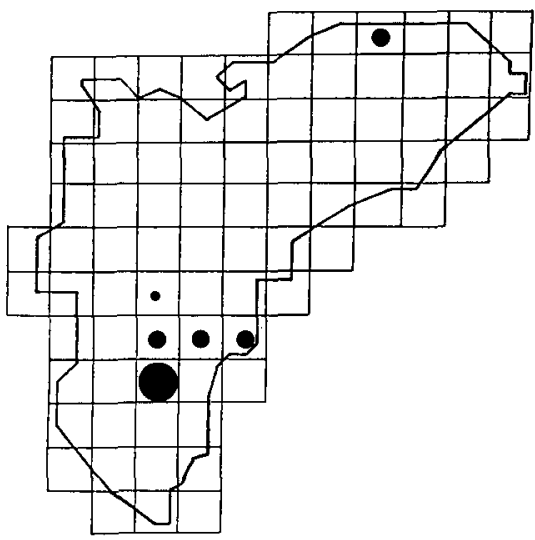

Escribano palustre

(Emberiza schoeniclus)

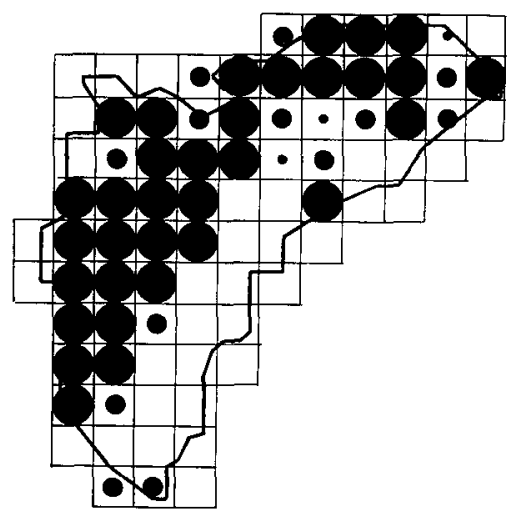

Escribano montesino (Emberiza cia)

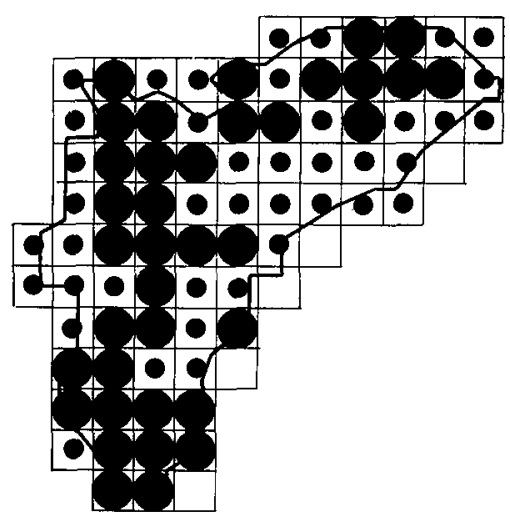

Triguero (Miliaria calandra)

\section{APÉNDICE II}

Especies que componen cada rama del dendrograma de la figura 6 al nivel 0.7, distribuidas según los 11 grupos comentados en el texto.

\section{Grupo I}

I.1: Tachybaptus ruficollis, Podiceps cristatus, Podiceps nigricollis, Ixobrichus minutus, Nycticorax nycticorax, Ardeola ralloides, Bubulcus ibis, Egretta garzetta, Ardea purpurea, Tadorna tadorna, Anas strepera, Anas platyrhinchos, Anas clypeata, Marmaronetta angustirrostris, Netta rufina, Ayrhya ferina, Circus aeruginosus, Rallus aquaticus, Porzana pusilla, Fu- 
lica atra, Gallinula chloropus, Himantopus himantopus, Recurvirostra avosetta, Glareola pratincola, Charadrius alexandrinus, Tringa totanus, Gelochelidon nilotica, Sterna hirundo, Sterna albifrons, Chlidonias hybrida, Alcedo atthis, Motacilla flava, Locustella luscinioides, Acrocephalus melanopogon, Acrocephalus scirpaceus, Acrocephalus arundinaceus, Panurus biarmicus, Remiz pendulinus, Emberiza schoeniclus.

I.2: Cettia rapiaria.

I.3: Riparia riparia.

I.4: Larus ridibundus, Larus argentatus.

I.5: Cisticola juncidis.

I.6: Coracias garrulus.

Grupo II

II.1: Falco tinnunculus, Alectoris rufa, Streptopelia turtur, Cuculus canorus, Athene noctua, Apus apus, Upupa epops, Galerida cristata, Hirundo rustica, Delichon urbica, Saxicola torquata, Oenanthe hispanica, Turdus merula, Sylvia melanocephala, muscicapa striata, Parus major, Lanius senator, Passer domesticus, Serinus serinus, Carduelis chloris, Carduelis carduelis, Emberiza cirlus, Miliaria calandra.

II.2: Sturnus unicolor.

Grupo III

III.1: Hieratus pennatus, Dendrocopos major, Lullula arborea, Erithacus rubecula, Sylvia communis, Phylloscopus bonelli, Regulus ignicapillus, $\mathrm{Ga}-$ rrulus glandarius.

III.2: Aegithalos caudatus, Parus ater, Certhia Brachydactyla.

III.3: Circaetus gallicus, Accipiter gentilis, Sylvia cantillans, Loxia curvirostra.

III.4: Strix aluco, Turdus viscivorus.

III.5: Accipiter nisus, Fringilla coelebs.

Grupo IV

IV.1: Aquila chrysaetos, Columba palumbus, Galerida teklae, Troglodytes troglodytes, Paryus cristatus, Corvus corax.

IV.2: Pyrrhocorax pyrrhocorax.

IV.3: Luscinia megarhynchos, Hippolais polyglotta.

IV.4: Hieraetus fasciatus, Falco peregrinnus, Oenanthe leucura, Monticola solitarius, Emberiza cia.

IV.5: Bubo bubo.

IV.6: Ptyonoprogne rupestris, Petronia petronia.

Grupo V

V.1: Hippolais pallida.

V.2: Sylvia hortensis.

Grupo VI

VI.1: Columba oenas, Caprimulgus ruficollis, Calandrella cinerea, Alauda arvensis, Lanius excubitor, Corvus monedula. 
VI.2: Buteo buteo, Coturnix coturnix, Pica pica, Carduelis cannabina.

VI.3: Clamatur glandarius.

VI.4: Phoenicurus ochruros, Sylvia atricapilla.

VI.5: Tyto alba, Merops apiaster.

VI.6: Picus viridis.

VI.7: Motacilla alba.

VI.8: Sylvia undata, Oriolus oriolus.

Grupo VII

VII.1: Corvus corone.

VII.2: Columba livia.

VII.3: Cercotrichas galactotes.

Grupo VIII

VIII.1: Charadrius dibius, Vanellus vanellus.

VIII.2: Passer montanus.

VIII.3: Apus melba.

Grupo IX

IX.1: Calandrella rufescens, Sylvia conspicillata.

IX.2: Circus pygargus, Burhinus oedicnemus.

IX.3: Falco subbuteo, Tetrax tetrax, Pterocles orientalis.

IX.4: Otus scops, Asio otus, Melanocorhypha calandra.

IX.5: Actitis hypoleucos.

Grupo X

X.1: Phalacrocorax aristotelis, Pandion haliaetus, Apus pallidus.

$\mathrm{X} .2$ : Jynx torquilla.

Grupo XI

XI.1: Hirundo daurica.

XI.2: Motacilla cinerea.

\section{ADDENDUM}

Una vez finalizado el trabajo y en proceso de impresión, J. Navarro y L. Rico detectaron la nidificación de una nueva especie en la provincia, el Cernícalo Primilla (Falco naumanni), en la cuadrícula XY8060. 